\title{
Galactic Center gas clouds and novel bounds on ultralight dark photon, vector portal, strongly interacting, composite, and super-heavy dark matter
}

\author{
Amit Bhoonah, ${ }^{1}$ Joseph Bramante, ${ }^{1,2}$ Fatemeh Elahi, ${ }^{3}$ and Sarah Schon ${ }^{1,2}$ \\ ${ }^{1}$ The McDonald Institute and Department of Physics, Engineering Physics, \\ and Astronomy, Queen's University, Kingston, Ontario, K7L 2S8, Canada \\ ${ }^{2}$ Perimeter Institute for Theoretical Physics, Waterloo, Ontario, N2L 2Y5, Canada \\ ${ }^{3}$ School of Particles and Accelerators, Institute for Research in Fundamental Sciences IPM, Tehran, Iran
}

(Received 11 January 2019; published 8 July 2019)

\begin{abstract}
Cold gas clouds recently discovered hundreds of parsecs from the center of the Milky Way Galaxy have the potential to detect dark matter. With a detailed treatment of gas cloud microphysical interactions, we determine Galactic Center gas cloud temperatures, unbound electron abundances, atomic ionization fractions, heating rates, and cooling rates and find how these quantities vary with metallicity. Considering a number of different dark sector heating mechanisms, we set new bounds on ultralight dark photon dark matter for masses $10^{-22}-10^{-10} \mathrm{eV}$, vector portal dark matter coupled through a sub-mega electron volt mass boson, and up to $10^{60} \mathrm{GeV}$ mass dark matter that interacts with baryons.
\end{abstract}

DOI: 10.1103/PhysRevD.100.023001

\section{INTRODUCTION}

Although its gravitational influence has been observed in galactic and cosmological dynamics, dark matter's nongravitational couplings and cosmological origin remain unknown. Discovering dark matter's features would increase our knowledge of the structure and history of the physical Universe. In this work, we determine how cold gas clouds near the center of the Milky Way galaxy can be used to detect dark matter.

Some prior work has considered the impact dark matter can have on interstellar and intergalactic gas [1-3]. The physical basis of these studies is remarkably simple: dark matter tends to have a higher temperature than the coldest interstellar and intergalactic gas. Therefore, dark matter, which is often more dense than diffuse astrophysical gas, can heat this gas to higher-than-observed temperatures, if dark matter interacts enough with baryons or electrons in the gas cloud. A somewhat distinct mechanism for dark matter heating of interstellar gas was more recently identified in Ref. [4]. A long-range and slowly shifting electric potential will heat interstellar gas by transforming electromagnetic potential energy into gas kinetic energy, through the acceleration and collision of gas particles. Such a long-range oscillating electric potential is sourced by vectorlike dark matter with a very long Compton

Published by the American Physical Society under the terms of the Creative Commons Attribution 4.0 International license. Further distribution of this work must maintain attribution to the author(s) and the published article's title, journal citation, and DOI. Funded by SCOAP . wavelength and a small mixing with the photon of the Standard Model, known as ultralight dark photon dark matter. Most recently, in Ref. [3], the authors of this paper identified that cold gas clouds near the Galactic Center provide unprecedented sensitivity to dark matter capable of heating interstellar gas. In the following work, the best bounds are set by the coldest cloud, denoted G1.4 - 1.8 +87 . We want to highlight here the concern that for this particular object the temperature measurement is the result of a single-channel fluctuation and therefore the true temperature may be considerably hotter than $22 \mathrm{~K}$. The cloud is nonetheless included, albeit marked as "preliminary" to indicate its status. We strongly encourage followup observations to confirm relevant properties as well as to motivate future searches for these types of cold gas clouds. For further discussion, see Sec. VII.

A few hundred cold gas clouds were recently discovered, each situated a few hundred parsecs from the center of the Milky Way Galaxy [5]. Based on their speed, it seems likely that these gas clouds were formed tens of millions of years ago in the central molecular zone, before being pushed by a Galactic wind out of the core of the Galaxy. These gas clouds provide unparalleled sensitivity to dark matter with certain interactions, as explored in Ref. [3]. Specifically, cold gas clouds provide a physical environment unobtainable by terrestrial experiments; cold gas clouds contain hundreds of solar masses of ionized gas, which is rather sensitive to heating by dark matter with relatively strong couplings to baryons, or dark matter with a long-range interaction with electrons. One example of the latter is dark matter with a small electromagnetic charge, often called millicharged dark matter. In Ref. [3], cold 
Galactic Center gas clouds placed leading constraints on dark matter strongly coupled to baryons and millicharged dark matter.

Here, we study the properties of Galactic Center gas clouds in more detail using a numerical code and derive additional bounds on dark matter models. The remainder of this paper proceeds as follows. In Sec. II, we review gas cloud physics and study the properties of gas clouds discovered near the Galactic Center, modeling their thermal properties using the numerical code CLOUDY. A new bound on ultralight dark photon dark matter is obtained in Sec. III. The sensitivity of Galactic Center gas clouds to vector portal dark matter coupled through a sub-kilo electron volt mass mediator is shown in Sec. IV. New bounds on dark matter which interacts with baryons through a spin-independent coupling are found in Sec. V along with the first derivation of the gas cloud "overburden"; it is found that gas cloud bounds apply to dark matter as massive as approximately $10^{60} \mathrm{GeV}$. In Sec. VI, we conclude with some discussion of how the temperature profile of gas clouds might be used to discover dark matter.

\section{GALACTIC CENTER GAS CLOUD PROPERTIES}

In a previous paper [3], we showed that cold, atomic gas clouds near the Galactic Center provide a unique testing ground for a number of dark matter models. That work utilized simple volumetric interstellar gas cooling rates we obtained from Ref. [6]. However, the physics governing the thermal, ionization, and chemical state of this intergalactic gas is complex. Here, we initiate a fuller treatment of Galactic Center gas cloud dynamics. Specifically, we make use of the gas microphysics code CLOUDY, last described in Ref. [7], to simulate objects matching the physical properties of the clouds observed by McClure-Griffiths et al. [5] used in our prior analysis. These simulations yield improved ionization and cooling rates, which are essential to accurately determine cold gas cloud sensitivity to dark matter models.

\section{A. ISM and gas physics}

The study of low-density interstellar medium (ISM) atomic gas dynamics is an active area of research. Understanding the composition and thermal properties of the ISM is critical to understanding the formation and evolution of galaxies and stars. There are a number of processes that play key roles in governing the state and evolution of the ISM. For our purposes, we will be focusing on the factors regulating the heating and cooling of the gas. We will particularly investigate how the composition of the gas clouds and variation of the incident radiation fields change the thermal balance within the cloud and subsequently the ionization fraction and cooling rates.
The bulk of the ISM is made of hydrogen and helium. As the stellar population matures, the gas is further enriched with metals (taken here in the astrophysical context to mean any nuclei heavier than helium) as well as grains and more complex molecules. These additional components facilitate some of the dominant heating and cooling mechanisms, while also contributing to the regulation of the chemical network, which has further consequences for the gas's internal thermal processes.

Metallicities within the Milky Way's ISM are determined in a number of ways. Optical emission lines from singly and doubly ionized oxygen, OII and OIII, can be used to constrain oxygen abundances within Galactic ionized hydrogen (HII) regions. Then, total metallicities are inferred assuming linear scaling between the two [8]. Using a similar method, observations of UV absorption lines can be used to constrain the abundance of a variety of elements and subsequently the total metallicity [9]. The spectra of stars can also constrain metallicities at the time of the star's formation, and the combination of these observations shows the existence of a large scale metallicity gradient across the Galaxy [10,11].

Grains (or dust) [12] encompass material ranging from simple molecules to complex particles up to approximately $0.3 \mu \mathrm{m}$ in size. The grain population is split almost equally between silicate minerals and carbonaceous material. While the dust component only makes up a tiny fraction of the total ISM, it has significant effect on the chemical composition and physical state of the gas. Moreover, it acts as an important observational tracer of the aforementioned ISM conditions. Dust grain interactions with the gas include [13] heating through photoelectric emission, formation of $\mathrm{H}_{2}$ molecules from emission off of grains, and coupling magnetic fields to the neutral gas.

The thermal balance of the ISM is determined by the heating sources and the cooling mechanisms available to the gas. Energy is injected from various background radiation fields including the UV background, cosmic-ray background, and nearby stellar objects. Important heating mechanisms include [14] heating by low energy cosmic rays, photoelectric heating by grains, photoelectric heating by photoionization of atoms and molecules, grain-gas thermal exchange, as well as hydrodynamic/magnetohydrodynamics heating and interstellar shocks, though the latter two are not included in this treatment. The most important processes for the calculation performed in this work are heating from cosmic rays and grains. Depending on the make-up of the gas, cooling can be facilitated by [15] metal line transitions, collisional ionization, Ly $\alpha$ photons, recombination, bremsstrahlung and molecular cooling. For the low-density environment found in the gas clouds at hand, the most relevant processes are collisional excitation and subsequent decay of various metal species. In particular, carbon, oxygen, and iron provide the dominant cooling terms at the temperatures of interest here [16-18]. 
Energy is injected into the cloud via a radiation background. For this calculation, we take into account both the cosmic UV/x-ray background $[19,20]$ as well as the cosmic-ray background [21,22]. Given these sources of energy in combination with the relevant heating and cooling mechanisms, different ISM regions are often labeled according to their thermal and chemical phases [23,24]. These phases are typically called the cold neutral, warm neutral, and hot ionized media. Additional names for phases include the warm ionized medium [25] and cold molecular phase [26]. For a further review of the topic, see Ref. [27]. However, factors such as turbulence, the inflow of gas, and supernova feedback can disrupt these distinct phases and complicate this picture of the ISM considerably [28]. Therefore, we will not attempt to label the thermal and chemical phases of our cold gas clouds, although for the most part, the gas clouds we study here would fall in the "cold neutral" category.

\section{B. CLOUDY models}

The CLOUDY code works by calculating equations of energy, mass, and charge conservation, in addition to the detail balance equation governing the density of (ionized) atomic species $n_{i}$,

$$
\begin{aligned}
\frac{\partial n_{i}}{\partial t} & =\sum_{j \neq i} n_{j} R_{j i}+\text { source }-n_{i}\left(\sum_{j i} R_{i j}+\text { sink }\right) \\
& =0\left[\mathrm{~cm}^{-3} \mathrm{~s}^{-1}\right],
\end{aligned}
$$

where $R_{i j}$ is the total rate at which species $i$ goes to $j$. Processes such as photo- and collisional ionization, recombinations, and charge exchange contribute to the "source" and "sink" portions of the above equation. The relevant references for the physical processes can be found in Refs. [7,29] as well as the CLOUDY documentation. The additional parameters specified in CLOUDY for each gas cloud model are listed below:

(i) Metallicity.-We assume the metallicity of the cloud scales with solar metallicity-although the numerical code we use does account for depletion of individual atomic species due to absorption onto dust grains. While the metallicity gradient across the disk of the Milky Way is relatively well documented, the environment within the Galactic Center is perhaps not as straightforward. References [30,31] both find stars with a range of metallicities within the Galactic bulge, which is indicative of both older, metal poor, as well as newer populations of stars being present. As we are working here under the assumption that our population of gas clouds originates from within this Galactic Center region, we will investigate both metal rich and poor in our gas cloud models. (ii) Dust grains.-We use the ISM appropriate dust grain model provided by the CLOUDY code $[32,33]$. It includes both silicate and graphite components and has a size distribution and abundance that matches observed dust grain properties. Some metals from the gas phase are depleted as they are incorporated into the grain component, in particular calcium, aluminium, titanium, and iron.

(iii) UV/x-ray background.-We use the UV/x-ray background model described by Haardt and Madau [34]. This includes contributions from quasars as well as the cosmic microwave background (CMB).

(iv) Cosmic-ray background.-Cosmic rays provide a critical source of heating and ionization of the neutral gas. Furthermore, within the CLOUDY framework, the inclusion of cosmic rays is critical to allow the code to maintain the chemistry network as the environment approaches lower temperatures at which the gas becomes molecular. The default background provided by the code is the model by Indriolo et al. [35] with a mean ionization rate of $2 \times 10^{-16} \mathrm{~s}^{-1}$; we note that the mean ionization rate indicates the fraction of atoms ionized per second in interstellar gas. Other authors (see Ref. [36] and references within), however, find more conservative rates as low as a $1-2 \times 10^{-18} \mathrm{~s}^{-1}$, though more recent measurements seem to favor a value closer to $10^{-16} \mathrm{~s}^{-1}$. In contrast. McCall et al. [37] find a mean ionization rate of $1.2 \times 10^{-15} \mathrm{~s}^{-1}$ along a galactic line of sight. We therefore consider a range of possible cosmic-ray background ionization rates for our gas cloud models.

(v) Density profile.-We assume a constant gas density for the cloud models presented here. While present surveys of these gas clouds reveal largely uniform gas cloud densities [5], it would be interesting to extend our calculations for nonuniform gas cloud densities in future work.

\section{Gas cloud models}

We here present CLOUDY outputs for the three coldest clouds found in the atomic hydrogen survey. Details of the clouds are given in Table 1. Columns detailing average temperature, density, and radius are taken from Ref. [5], while the metallicity, dust grain model, and ultraviolet and cosmic-ray backgrounds are settable parameters within CLOUDY. Since atomic transitions of "metal" (heavier than helium) elements provide the dominant cooling mechanism, we explore the effect of varying gas cloud metallicity. For each cloud, we consider one solar, one high, and one low metallicity model, respectively called $\mathrm{C} 1, \mathrm{C} 2$, and $\mathrm{C} 3$, for a uniform gas density. The clouds were modeled as constant density spheres, although experimenting with oblate spheroid geometries did not substantially change the result. For each gas cloud, we tune the ultraviolet and 
TABLE I. Summary of the different gas cloud models simulated using CLOUDY, which match the properties of clouds G1.4 $-1.8+87$, G357.8 $-4.7-55$, and G1.5 $+2.9+1.05$ from Ref. [5]. The average temperature $(\bar{T})$, gas cloud radius, and density $(\bar{\rho})$ are taken from McClure-Griffiths, while the metallicity relative to solar metallicity $\left(Z / Z_{\odot}\right)$, the presence of dust grains in the simulation, the UV photon background flux relative to the standard normalization described in the text, the cosmic-ray background ionization rate (CR), and the density profile parameter were parameters varied in the code. The average electron density $\left(\bar{n}_{e}\right)$ and average cooling (ave. cooling) rates are used for setting bounds on dark matter.

\begin{tabular}{|c|c|c|c|c|c|c|c|c|c|}
\hline $\begin{array}{l}\text { Dark matter } \\
\text { Model }\end{array}$ & $\bar{T}(\mathrm{~K})$ & Radius (pc) & $\bar{\rho}\left(\mathrm{cm}^{-3}\right)$ & $Z / Z_{\odot}$ & Grains & UV & $\mathrm{CR}\left(\mathrm{s}^{-1}\right)$ & $\bar{n}_{e}\left(\mathrm{~cm}^{-3}\right)$ & $\begin{array}{l}\text { Ave. cooling } \\
\left(\mathrm{erg} \mathrm{cm}^{-3} \mathrm{~s}^{-1}\right)\end{array}$ \\
\hline $\mathrm{C} 1-22$ & 22 & 8.2 & 0.29 & 1 & No & 0.1 & $1 \times 10^{-18}$ & $2.3 \times 10^{-4}$ & $1.9 \times 10^{-29}$ \\
\hline $\mathrm{C} 2-22$ & 22 & 8.2 & 0.29 & 0.1 & No & $1.9 \times 10^{-3}$ & $1.9 \times 10^{-19}$ & $9.7 \times 10^{-5}$ & $1.6 \times 10^{-30}$ \\
\hline C3-22 & 22 & 8.2 & 0.29 & 5 & No & 0.1 & $5 \times 10^{-18}$ & $5.6 \times 10^{-4}$ & $6.2 \times 10^{-28}$ \\
\hline C1-137 & 137 & 12.9 & 0.421 & 1 & Yes & 1 & $5 \times 10^{-17}$ & $1 \times 10^{-3}$ & $3.4 \times 10^{-28}$ \\
\hline C2-137 & 137 & 12.9 & 0.421 & 0.1 & Yes & 1 & $3 \times 10^{-18}$ & $5 \times 10^{-4}$ & $8.2 \times 10^{-29}$ \\
\hline C3-137 & 137 & 12.9 & 0.421 & 5 & Yes & 1 & $1.9 \times 10^{-16}$ & $6.2 \times 10^{-3}$ & $6.1 \times 10^{-27}$ \\
\hline C1-198 & 198 & 12.3 & 1.57 & 1 & Yes & 1 & $2.9 \times 10^{-16}$ & $1.2 \times 10^{-2}$ & $2.4 \times 10^{-26}$ \\
\hline C2-198 & 198 & 12.3 & 1.57 & 0.1 & Yes & 1 & $1.1 \times 10^{-16}$ & $7.4 \times 10^{-3}$ & $8.2 \times 10^{-27}$ \\
\hline C3-198 & 198 & 12.3 & 1.57 & 5 & Yes & 1 & $1.4 \times 10^{-15}$ & $4.5 \times 10^{-2}$ & $1.5 \times 10^{-25}$ \\
\hline
\end{tabular}

cosmic-ray parameters (which will physically tend to vary depending on the location of the cloud) to produce a cloud matching the observed average temperature.

Two of the two key quantities required for setting dark matter bounds are the electron number density and the cooling rate. These are listed in the last two columns of Table 1 and presented in Fig. 1. The three rows in Fig. 1 correspond to the three gas clouds modeled, listed from coldest to hottest from top to bottom. Columns from left to right show the equilibrium cooling rate (assuming only standard astrophysical sources), temperature, and electron densities for the gas cloud models previously mentioned. Note that the $\mathrm{x}$ axis shows the depth into the cloud from the illuminated surface rather than the radius from the center of the object. In each plot, the blue solid, yellow dashed, and yellow dotted lines correspond to models C1, C2, and C3 (summarized in Table 1), respectively; these models assume different gas cloud metallicities as indicated. In the density plots, the solid black line indicates the uniform gas density. The other curves show the electron number density of the corresponding cloud models.

As expected, models with higher metallicities provide more efficient cooling channels and therefore allow for a greater external energy input, whether it be from standard astrophysical sources or dark matter, to maintain the temperature observed in Ref. [5]. Higher metallicity clouds also result in higher electron number densities because the metal species provide more readily ionized electrons to the gas. When setting bounds using these systems, it is important to note that both the electron density and cooling rate are not derived independently, and there are some uncertainties in both the metallicity, dust grain and molecular content, as well as the local UV radiation and cosmic-ray background. Follow-up observations may allow these to be constrained further and therefore provide improved dark matter bounds.

\section{Gas cloud bounds on dark matter}

In the following sections, we will present Galactic Center gas cloud bounds on dark matter. We note that these bounds are based on the fact that cold gas clouds cool predominantly via radiative cooling. As was pointed out in Ref. [3], radiative cooling for gas clouds with temperatures approximately $10-1000 \mathrm{~K}$ is a monotonic decreasing function of temperature (see, e.g., Fig. 4 in Ref. [38] and Fig. 5 in Ref. [6]). As a consequence of the monotonic decreasing form of gas cloud radiative cooling curves and the Second Law of Thermodynamics, there is a maximum possible heating rate for any gas cloud observed at fixed density and temperature ranging from approximately 10 to $1000 \mathrm{~K}$. This implies the following bound on dark matter heating of cold gas clouds,

$$
\text { VDHR } \leq \mathrm{VCR},
$$

where the first term is the volumetric heating of the gas cloud by dark matter and the second term is the volumetric cooling via radiative processes detailed earlier.

Because it is most likely that Galactic Center gas clouds have solar metallicity [39], especially given the relatively young age of the gas clouds in question (approximately $10 \mathrm{Myr}$ ), we use the solar metallicity model $\mathrm{C} 1$ for our gas clouds throughout the remainder of this paper to set bounds on dark sectors. As noted in Ref. [3], models C2 and C3 yield similar bounds in the case of dark matter that predominantly interacts with electrons or iron, which is the case for dark matter considered in all three sections of this paper. This is because the dark matter bounds on 

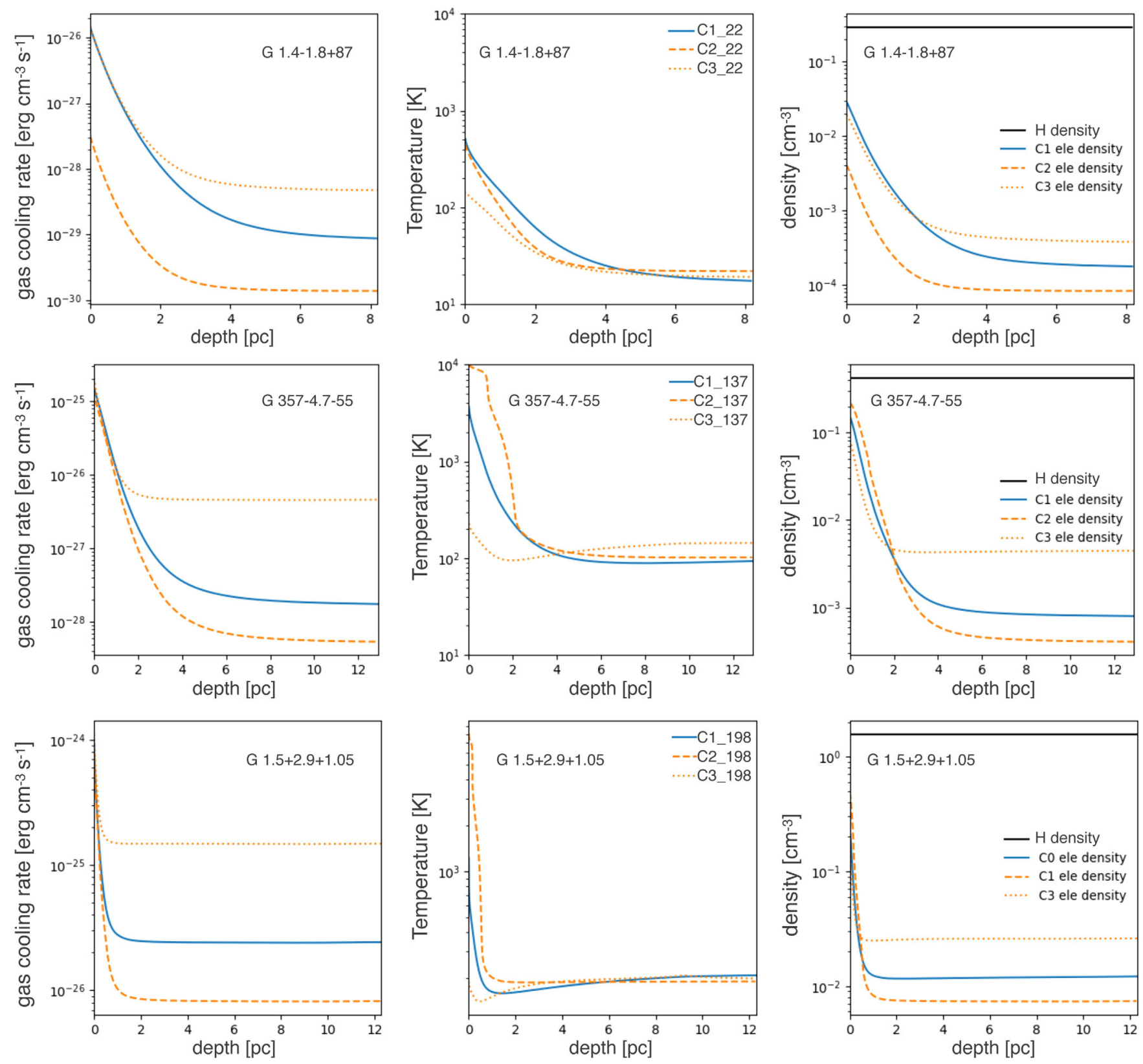

FIG. 1. CLOUDY models from top to bottom for clouds G1.4 $-1.8+87$, G357.8 $-4.7-55$ and G1.5 $+2.9+1.05$ from [5]. Columns from left to right show the equilibrium cooling rate, temperature, and unbound electron (and hydrogen) number density for a given radial depth into the cloud, as measured from the surface. As detailed in Table 1, the radial depth of these clouds varies from 8-12 parsecs. The solid blue, dashed and dotted orange lines correspond to the $\mathrm{C} 1, \mathrm{C} 2$, and $\mathrm{C} 3$ gas cloud models described in Table 1 . In the density plots, the solid black line shows the constant hydrogen density.

interaction cross sections scale linearly with both the gas cloud cooling rate and the electron or iron density. On the other hand, as can be verified from Table 1, the gas cloud cooling rate scales inversely with gas cloud metallicity (and by extension, iron density), and the gas cloud cooling rate also scales inversely with electron density. This makes the gas cloud heating bounds on dark matter interactions relatively insensitive to assumptions about gas cloud metallicity.
A few comments are in order concerning the dark matter density in the Galactic Center, as this does affect bounds on dark matter interactions with Galactic Center gas clouds. Technically, there is no direct evidence of dark matter in the central $3 \mathrm{kpc}$ of the Milky Way, since this region is predominantly composed of baryonic matter [40,41]. However, results from N-body simulations, hierarchical clustering, and dynamical halo structure considerations [42] indicate that the most plausible halos will have a 
shape similar to the eponymous Navarro-Frenk-White (NFW) profile [43]. In this study, we will use a generalized NFW profile as presented in Ref. [41],

$$
\rho_{x}(r)=\rho_{0}\left(\frac{r_{0}}{r}\right)^{\gamma}\left(\frac{r_{s}+r_{0}}{r_{s}+r}\right)^{3-\gamma},
$$

where $r_{s}=20 \mathrm{kpc}$ is the standard scale radius of the Milky Way. We will use the generalized NFW paramaters in Ref. [41], which were fit to match a morphological model of the stellar matter in the inner "bulge" region of the Milky Way galaxy [44]. The parameters of model "CjX" in Ref. [41] are $r_{0}=8 \mathrm{kpc}, \gamma \approx 1.03$, and $\rho_{0}=0.471 \mathrm{GeV} / \mathrm{cm}^{3}$. This yields dark matter densities near Galactic Center gas clouds of approximately $\rho \sim 10 \mathrm{GeV} / \mathrm{cm}^{3}$, which agrees well with standard halo profile parameters in the literature [3]. In the captions of bounds presented in this paper, we provide a simple prescription to rescale bounds, for readers who wish to consider the effect of different background dark matter densities.

The line-of-sight distances of gas clouds G1.4-1.8+87, G357.8 - 4.7-55, and G1.5 + $2.9+1.05$ from the Galactic Center are $R_{G 1.4}=0.31 \mathrm{kpc}, \quad R_{G 357}=0.75 \mathrm{kpc}$, and $R_{G 357}=0.41 \mathrm{kpc}$, respectively. Because the generalized NFW halo model predicts an increased dark matter density in the Galactic Center, when calculating Galactic Center gas cloud local dark matter densities, we will conservatively multiply these line-of-sight distances by a factor of $\sqrt{2}$ to account for their projected distance from the Galactic Center. Therefore, the projected distances we use for G1.4-1.8+87, G357.8-4.7-55, and $\mathrm{G} 1.5+2.9+1.05$ are $r_{G 1.4}=0.44 \mathrm{kpc}, r_{G 357}=1.1 \mathrm{kpc}$, and $r_{G 357}=0.58 \mathrm{kpc}$, respectively, implying dark matter densities near these three gas clouds of $\rho_{x, G 1.4}=17 \mathrm{GeV} / \mathrm{cm}^{3}$, $\rho_{x, G 357}=6.6 \mathrm{GeV} / \mathrm{cm}^{3}, \quad$ and $\rho_{x, G 1.4}=13 \mathrm{GeV} / \mathrm{cm}^{3}$, respectively.

Finally, we note that, throughout this document, we will use a Galactic Center velocity dispersion of $\bar{v} \approx 180 \mathrm{~km} / \mathrm{s}$. This velocity dispersion is consistent with results in Ref. [40] and is on the low end of velocity dispersion values allowed for by Milky Way dynamical considerations [41]. Using this velocity dispersion will tend to produce conservative bounds in the case of dark matter-nucleon scattering and dark matter-electron scattering for heavy dark photon mediated dark matter (for which the dark matter induced gas cloud heating rate scales roughly as velocity cubed). On the other hand, this low velocity dispersion does produce slightly aggressive bounds in the case of very light dark photon mediated dark matter, considered at the beginning of Sec. IV (for which dark matter induced gas cloud heating scales inversely with velocity). In the latter case, we have verified that changing the velocity dispersion by a factor of 2 changes the bounds on the y axis coupling parameters in Fig. 3 by less than a factor of 1.2, which is not visible on the scale of the plot.

\section{ULTRALIGHT DARK PHOTON DARK MATTER}

Ultralight dark photon dark matter requires a rather simple extension of the Standard Model (SM), where the Standard Model gauge group is augmented by an extra local $U(1)$ symmetry. This model has, in addition to the Standard Model hypercharge, another Abelian gauge boson, which we denote by $A^{\prime}$ and call the "dark photon" [45-51]. The dark photon has a mass and a kinetic mixing with the Standard Model hypercharge boson. For dynamical processes occurring in the sub-giga electron volt range, the physical $A^{\prime}$ field mixes predominantly with the Standard Model photon. The resulting Lagrangian is

$$
\mathcal{L}=\mathcal{L}_{\mathrm{SM}}-\frac{1}{4} F_{\mu \nu} F^{\mu \nu}-\frac{1}{4} F_{\mu \nu}^{\prime} F^{\prime \mu \nu}+m^{2} A_{\mu}^{\prime} A^{\prime \mu}-\frac{e}{(1+\epsilon)^{2}}\left(A_{\mu}+\epsilon A_{\mu}^{\prime}\right) J_{\mathrm{EM}}^{\mu},
$$

Here, the kinetic part of the Lagrangian has been diagonalized, and we have adopted the convention of Ref. [4] for the definition of the mixing parameter $\epsilon$. The interested reader is invited to consult Ref. [52] for a review of the form of Lagrangians with two local $U(1)$ gauge symmetries. The mass $m$ of the dark photon can be generated via the Stückelberg mechanism for simplicity, although it is straightforward to add an extra scalar field and generate $m$ via spontaneous symmetry breaking. The interaction part of this Lagrangian consists of the electromagnetic current $J_{\mathrm{EM}}^{\mu}$ coupled to the photon and the dark photon, with the latter coupling suppressed by a factor of $\epsilon$ in the limit that $\epsilon \ll 1$.
Much like axion dark matter, an ultralight dark photon is a plausible dark matter candidate because it can provide a matterlike energy density via oscillations of the dark photon field. Ultralight dark photon dark matter can be produced by cosmological excitation of its longitudinal or transverse field components as first considered in Refs. [53-55]. Assuming no additional couplings to lighter fields, ultralight dark photon dark matter is metastable, since for $m_{A^{\prime}} \ll 2 m_{e}$, it decays to three photons with a rather long lifetime [56]

$$
\tau_{A^{\prime}}=\frac{2^{7} 3^{6} 5^{3} \pi^{3}}{17 \epsilon^{2} \alpha_{\mathrm{EM}}^{4} m_{e}}\left(\frac{m_{e}}{m_{A^{\prime}}}\right)^{9} .
$$


It can be verified that, even for an order 1 mixing, $\epsilon \sim O(1)$, so long as $m \ll \mathrm{keV}$, the dark photon is long lived enough to be a dark matter candidate. Recently, a number of studies have indicated that an excited scalar field can transfer energy to a light dark photon field and that through this mechanism even extremely light dark photons may constitute the entire dark matter abundance [57-59].

In this work, we place new bounds on ultralight dark photon dark matter for $m \leq 10^{-10} \mathrm{eV}$. We use the heating mechanism detailed in Ref. [4], which can be summarized as follows. An ultralight dark photon, through its mixing with the Standard Model, photon produces an oscillating electric field which generates a current and dissipation in any medium that is not a perfect conductor. In our case, cold gas clouds at the Galactic Center harbor unbound electrons and ions which will collide with each other after they are accelerated by the oscillating electric field. This altogether transforms dark photon potential energy into the kinetic energy of charged particles in cold gas clouds. We will only highlight the most important facets of the mechanism here-the interested reader is encouraged to consult Ref. [4] for a thorough derivation of the effect.

We model the ionized part of our gas clouds as a nonrelativistic plasma. For the purpose of setting bounds on dark photon dark matter, this is a conservative approximation, since we neglect collisions between the ionized and neutral component of our gas clouds, where these additional collisions would result in greater energy transferred from the dark photon field to the gas. For gas clouds reported in Ref. [5], the plasma frequency, i.e., the typical electrostatic oscillation frequency of electrons in response to a charge separation, is

$\omega_{p}=\sqrt{\frac{4 \pi n_{e}}{m_{e}}} \approx 5 \times 10^{-13} \mathrm{eV}\left(\frac{n_{e}}{2 \times 10^{-4} \mathrm{~cm}^{-3}}\right)^{1 / 2}$,

where here we normalize to gas cloud G1.4 $-1.8+87$ 's electron density $n_{e} \approx 2 \times 10^{-4} \mathrm{~cm}^{-3}$, as given in Table 1 .

Since we are considering a plasma medium and not empty space, there is a screening effect that limits the interaction range of electrons with any external electric field. This is the Debye length of the plasma $\left(\lambda_{\mathrm{d}}=\sqrt{T_{\mathrm{g}} /\left(4 \pi \alpha_{\mathrm{EM}} n_{\mathrm{e}}\right)}\right)$, which can be interpreted as the effective Compton wavelength (related to the effective mass) of the dark photon in a plasma. If the dark photon mass is around the size of the plasma frequency, $m \sim \omega_{p}$, the dark photon can resonantly convert into ordinary photons. Because of this dark photon conversion process, cosmic microwave background data can be used to exclude dark photons with masses in excess of approximately $10^{-14} \mathrm{eV}$ [60]. However, for masses below this, dark photons will not decay to photons in the early Universe, and gas cloud heating by dark photon dark matter places leading bounds on this region of parameter space.

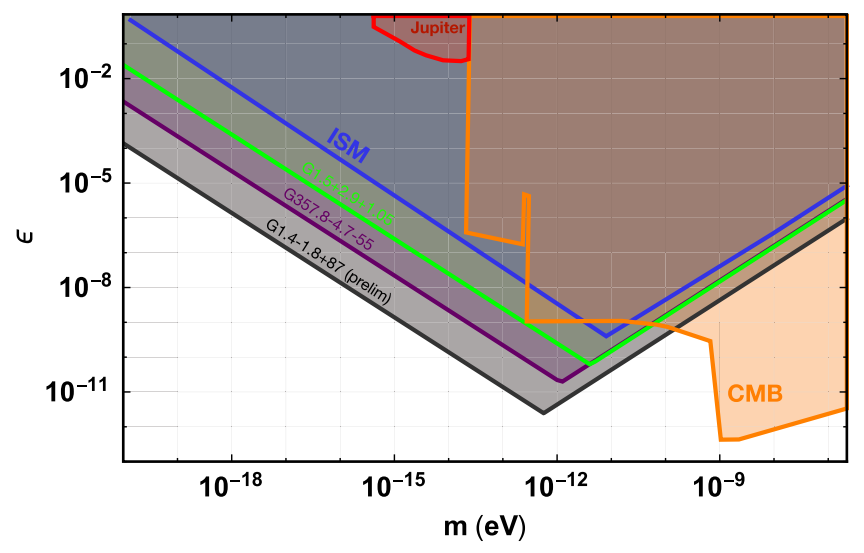

FIG. 2. Bounds on ultralight dark photons with mass $m$ and kinetic mixing parameter $\epsilon$, using three cold Galactic Center gas clouds, with parameters given in Table 1, and local dark matter densities according to a generalized NFW profile and projected distances from the Galactic Center detailed at the end of Sec. II. Readers wishing to rescale these bounds for different dark matter background density models should note that the bound on $\epsilon$ scales as $\frac{1}{\rho_{x}^{2}}$. Bounds using gas cloud $G 1.4-1.8+87$, using the temperature reported in Ref. [5], have been indicated as preliminary; see Sec. VII. Different CMB limits from the decay of dark photons into Standard Model photons are also shown in orange [60]. Bounds from satellite measurements of Jupiter's magnetic field are given in red [60]. A constraint from heating of the Milky Way's interstellar medium by dark photons is shown in blue [4]. The same mechanism, but applied to gas clouds with average temperature $137 \mathrm{~K}$ (G357.8 - 4.7 - 55 with an average cooling rate of $\left.3.4 \times 10^{-} 28 \mathrm{erg} \mathrm{s}^{-1} \mathrm{~cm}^{-3}\right)$ and $22 \mathrm{~K}(\mathrm{G} 1.4-1.8+87$ with an average cooling rate of $\left.1.9 \times 10^{-29} \mathrm{erg} \mathrm{s}^{-1} \mathrm{~cm}^{-3}\right)$ are shown in purple and black, respectively.

To obtain the heating rate due to the ultralight dark photon field, the equations of motion of the two $U(1)$ vector fields are solved and combined with that of the nonrelativistic plasma to obtain the frequency of the dark photon modes, $\omega$. The latter has a real and an imaginary part, $\omega=\omega_{h}+i \gamma_{h}$, and the imaginary part, $\gamma_{h}$, gives the volumetric heating rate $Q$, i.e., the heating rate per unit volume

$$
Q=2\left|\gamma_{h}\right| \rho_{x}
$$

where $\rho_{x}$ is the dark matter density. In our case, the dark matter density is $\rho_{x} \sim 10 \mathrm{GeV} \mathrm{cm}^{-3}$ for cold gas clouds near the Galactic Center [3]. The asymptotic expressions for $\gamma_{h}$ in the limit that the dark photon mass is either smaller or larger than the gas cloud plasma frequency are

$$
\gamma_{h}= \begin{cases}-\frac{\nu}{2}\left(\frac{m}{\omega_{p}}\right)^{2} \frac{\epsilon^{2}}{1+\epsilon^{2}}, & m \ll \omega_{p} \\ -\frac{\nu}{2}\left(\frac{\omega_{p}}{m}\right)^{2} \frac{\epsilon^{2}}{1+\epsilon^{2}}, & m \gg \omega_{p},\end{cases}
$$

where $\nu$ is the collision frequency describing the interaction of electrons and ions in the plasma, 


$$
\nu=\frac{4 \sqrt{2 \pi} \alpha_{\mathrm{EM}}^{2} n_{e}}{3 \sqrt{m_{e} T^{3}}} \log (\Lambda) .
$$

Here, $\alpha_{\mathrm{EM}}$ is the electromagnetic fine structure constant, and $\Lambda$ is the Coulomb logarithm, $\log (\Lambda)=\log \sqrt{\frac{4 \pi T^{3}}{\alpha_{\mathrm{EM}}^{3} n_{e}}}$. Even though the expressions for the heating rate in (8) are asymptotic, we take them as hard cutoffs in establishing our bounds for light dark photons shown in Fig. 2. Further work would need to be done to accurately model dark photon heating of the plasma when $\omega_{p} \sim m$. We display new bounds on ultralight dark photon dark matter, obtained by equating the heating rate give in Eq. (8) to the cooling rates of gas clouds G357.8-4.7-55 and G1.4 - 1.8 + 87, as given in Table 1 .

\section{SUB-MEGA ELECTRON VOLT MEDIATOR VECTOR PORTAL DARK MATTER}

Besides being a dark matter candidate, dark photons can also serve as the mediator between dark matter and the SM. Indeed, this scenario has been extensively studied in the last decade [46,47,61-66]. Cold Galactic Center gas clouds will prove especially sensitive to vector portal dark matter coupled through a sub-mega electron volt mass dark photon, with intermediate strength couplings. Such dark matter evades detection by terrestrial experiments, because it is moving too slowly to be detected after scattering with the Earth's atmosphere and crust, to excite electrons to detectable energies in existing experiments.

We will consider a simple vector portal model to demonstrate that cold gas clouds can be used to explore dark matter models with light mediators. Our results indicate that other dark matter models coupled to the Standard Model through light mediators may also be constrained by cold Galactic Center gas clouds; this is left to future work. Here, we take dark matter to be a Dirac fermion $\chi$ that communicates with the Standard Model via a kinetically mixed dark photon $A^{\prime}$. Specifically, the Lagrangian we will be studying is the following:

$\mathcal{L}=\mathcal{L}_{\mathrm{SM}}-\frac{1}{2} m_{A^{\prime}}^{2} A_{\mu}^{\prime} A^{\prime \mu}-\frac{1}{4} F_{\mu \nu}^{\prime} F^{\prime \mu \nu}-\frac{\kappa}{2} F_{\mu \nu} F^{\prime \mu \nu}-g_{D} A_{\mu}^{\prime} \bar{\chi} \gamma^{\mu} \chi$.

We note that in this section we will denote the kinetic mixing parameter as $\kappa$, in keeping with historical convention [46] and to not confuse this with the $\epsilon$ mixing parameter in Sec. III; note that $\epsilon$ and $\kappa$ have different definitions. To take this gauge basis Lagrangian to the mass basis, we shift $A_{\mu} \rightarrow A_{\mu}-\kappa A_{\mu}^{\prime}$, which results in an electromagnetic current for $A^{\prime}$ with a coupling proportional to $\kappa$. The parameters in this simplified vector portal model are the dark photon mass $\left(m_{A^{\prime}}\right)$, the dark matter mass $\left(m_{\chi}\right)$, the gauge coupling of the dark photon with dark matter $\left(g_{D}\right)$, and $\kappa$, which determines the coupling of dark photon with Standard Model fields.
Since the interactions of the dark photon proceed through the Standard Model photon current, vector portal dark matter interacts with Standard Model particles charged under $U(1)_{\mathrm{EM}}$. For the coldest Galactic Center gas cloud of interest, G1.4 - 1.8 +87 , we will only consider dark matter interactions with unbound electrons in the cloud; interactions with electrons bound to atoms and nuclei will be of subdominant importance because the energy deposited on unbound electrons will be much larger. For the model detailed above, the cross section for dark matter scattering with unbound electrons has the following form,

$$
\sigma_{\chi e}=\frac{8 \pi \kappa^{2} \alpha_{D} \alpha_{\mathrm{EM}} \mu_{\chi e}^{2}}{\left(m_{A^{\prime}}^{2}+q^{2}\right)^{2}}
$$

with $\mu_{\chi e} \equiv \frac{m_{\chi} m_{e}}{m_{\chi}+m_{e}}$ being the reduced mass of the dark matter (DM)-electron system, $\alpha_{D}=g_{D}^{2} /(4 \pi), \quad q=\sqrt{2 E_{\mathrm{nr}} m_{e}}$ being the momentum transfer between the dark matter and electron, and $E_{\mathrm{nr}} \approx \mu_{\chi e}^{2} v_{x}^{2} / m_{e}$ being the average energy transferred per elastic scattering interaction. Using cold gas clouds, a constraint on this model is obtained by requiring $\mathrm{VCR}>\mathrm{VDHR} \sim n_{e} n_{x} \sigma_{x} v_{x} E_{\mathrm{nr}}$, where VCR is the cooling rate of the designated gas cloud per unit volume and VDHR represents the DM heating rate per unit volume:

$\mathrm{VCR}>\frac{8 \pi \kappa^{2} \alpha_{D} \alpha_{\mathrm{EM}} n_{e} n_{x} \mu_{\chi e}^{4}}{m_{e}} \int d^{3} v_{x} v_{x}^{3} \frac{B\left(v_{x}, v_{\mathrm{esc}}, y\right)}{\left(m_{A^{\prime}}^{2}+2 \mu_{\chi e}^{2} v_{x}^{2}\right)^{2}}$.

The integral is taken from zero to the escape velocity of dark matter from the Milky Way, which is approximately 0.002 [67], with $y \equiv \cos \theta$ indicating the angle between the dark matter and the electron, and B is a Maxwellian DM velocity distribution defined in Ref. [3], with normalization $\int d^{3} v_{x} B=1$. To gain some intuition for the bounds provided by Galactic Center gas clouds over a range of dark photon masses $\left(m_{A^{\prime}}\right)$, we will investigate two limits: 1) $m_{A^{\prime}} \ll q$, in which the interaction of dark matter with electrons is through a long-range force, and 2) $m_{A^{\prime}} \gg q$, in which the interaction of dark matter with electrons is a contact interaction.

In the limit that $m_{A^{\prime}} \rightarrow 0$, we would expect an infrared divergence to arise in the integral in Eq. (11), since in the limit $v_{x} \rightarrow 0$ the integral diverges. This infrared divergence is regulated for dark matter scattering in cold gas clouds because the dark photon will gain a thermal mass through its interactions with the gas cloud plasma as also discussed in Sec. III. The thermal mass can be found from the Debye length, which is $\lambda_{\mathrm{d}}=\sqrt{T_{\mathrm{g}} /\left(4 \pi \alpha_{\mathrm{EM}} n_{\mathrm{e}}\right)}$. The Debye length indicates the scale at which the dark photon necessarily mediates finite-range interactions in a cold gas cloud, compared to infinite-range interactions it would mediate in empty space. Taking the thermal mass into consideration, we arrive at a well-defined infrared cutoff for the case of a very light $A^{\prime}$. For $m_{A^{\prime}} \ll q$, 


$$
\kappa^{2} \alpha_{D} \alpha_{\mathrm{EM}}<\frac{\mathrm{VCR}}{n_{e}}\left[\frac{2 \pi n_{x}}{m_{e}} \int d^{3} v_{x} \frac{B\left(v_{x}, v_{\mathrm{esc}}, y\right)}{v_{x}} \log \left(\frac{2 \mu_{\chi e}^{2} v_{x}^{2}}{\left(\max \left[1 / \lambda_{d}, m_{A^{\prime}}\right]\right)^{2}}\right)\right]^{-1}
$$

The millicharged DM is the special case of the scenario discussed here, where the vacuum mass of $A^{\prime}$ is zero. If $m_{A^{\prime}}>1 / \lambda_{d}$, then the infrared divergence is regulated by the mass of a dark photon.

When the dark photon is larger than both the plasma frequency and the maximum momentum exchanged between the electron and dark matter, the dark matter electron scattering interaction becomes a contact interaction. For $m_{A^{\prime}} \gg q, 1 / \lambda_{d}$,

$\kappa^{2} \alpha_{D} \alpha_{\mathrm{EM}}<\frac{\mathrm{VCR}}{n_{e}}\left[\frac{8 \pi n_{x} \mu_{\chi e}^{4}}{m_{e}} \int d^{3} v_{x} v_{x}^{3} \frac{B\left(v_{x}, v_{\mathrm{esc}}, y\right)}{m_{A^{\prime}}^{4}}\right]^{-1}$,

which is well defined in the sense that there are no divergences in this expression.

In Figs. 3 and 4, we plot galactic gas cloud constraints on vector portal models, using Eq. (11), gas cloud parameters given in Table 1, particularly the average volumetric cooling rate obtained for gas cloud G1.4-1.8+87, $\mathrm{VCR}=1.9 \times 10^{-29} \mathrm{ergs} \mathrm{cm}^{-3} \mathrm{~s}^{-1}$. In Fig. 3, we note that for the mass choice $m_{A^{\prime}}=10^{-7} \mathrm{GeV}$ the bound shifts dramatically at $m_{\chi}=10^{-4} \mathrm{GeV}$. This is because at this mass value the dark photon mass is roughly equal to the momentum exchanged between the dark matter and electron, $m_{A^{\prime}} \sim q \sim m_{\chi} v_{x}$. Put another way, at this dark matter mass, the dark matter-electron scattering dynamics shift from long-range to contact interactions; therefore, the relevant bound shifts from Eq. (12) to Eq. (13).

In Figs. 3 and 4 and the preceding treatment, we have considered relatively small couplings between dark matter and electrons. However, if the coupling of dark matter to electrons $\left(\kappa^{2} \alpha_{D} \alpha_{\mathrm{EM}}\right)$ is sufficiently large, bounds from Galactic Center gas clouds will no longer apply. Dark matter with a large enough coupling to electrons will deposit most of its kinetic energy into electrons near the surface of the gas cloud and may not appreciably heat the interior region. To estimate this effect, we will require that dark matter retain at least half of its initial kinetic energy by the time it reaches the center of the gas cloud. The energy transfer of a dark matter to the cloud is simply $d E / d r=n_{e} \sigma_{\chi e} E_{\mathrm{nr}}$. Given that the depth of the gas cloud G1.4-1.8+87 is $r=8 \mathrm{pc}$, the coupling for which dark matter loses half its kinetic energy after traveling the radius of the cloud is

$$
\kappa^{2} \alpha_{D} \alpha_{\mathrm{EM}} \sim 10^{-7}\left(\frac{m_{\chi}}{\mathrm{GeV}}\right)
$$

for a long-range interaction $\left(m_{A^{\prime}} \ll q\right)$, and

$$
\kappa^{2} \alpha_{D} \alpha_{\mathrm{EM}} \sim 1 \times\left(\frac{m_{\chi}}{\mathrm{GeV}}\right)\left(\frac{0.5 \mathrm{MeV}}{\mu_{\chi e}}\right)^{4}\left(\frac{m_{A^{\prime}}}{10 \mathrm{keV}}\right)^{4},
$$

for an interaction with $m_{A^{\prime}} \gg q$.
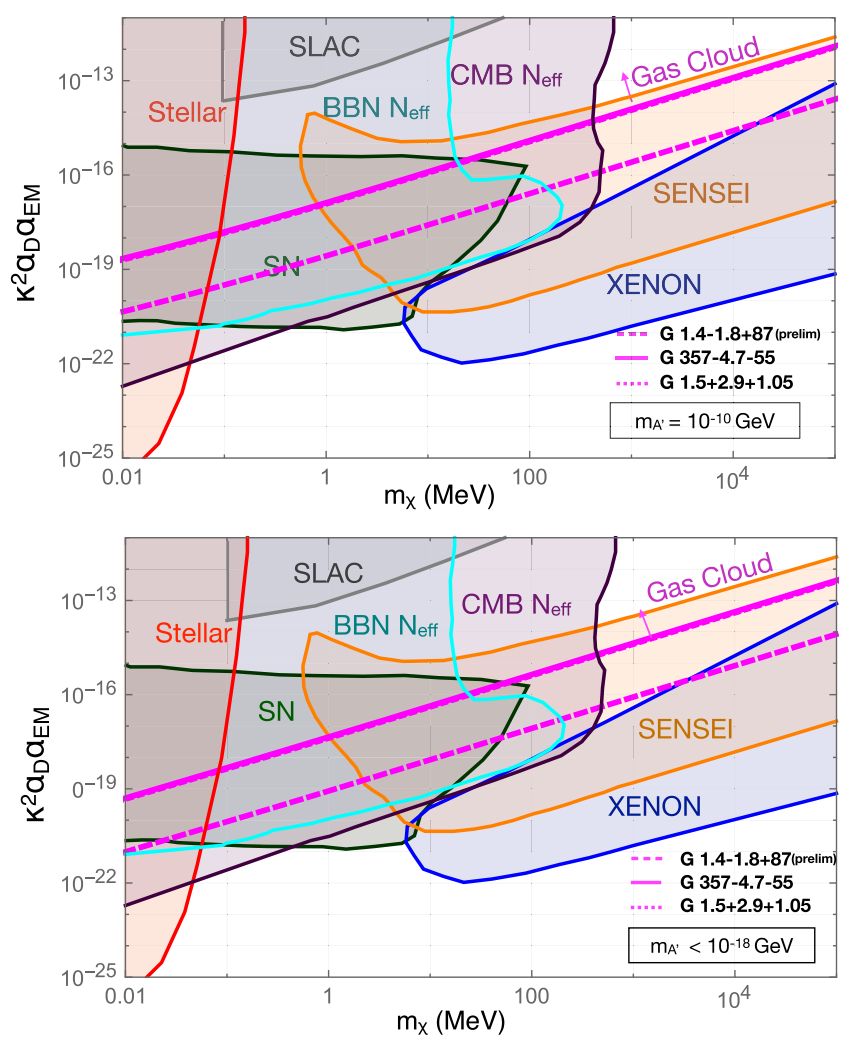

FIG. 3. The constraint on the product of the couplings $\kappa^{2} \alpha_{D} \alpha_{\mathrm{EM}}$ is shown in magenta (regions above each line are excluded), for vector portal dark matter heating of Galactic Center gas clouds with gas cloud parameters given in Table 1 and local dark matter densities according to a generalized NFW profile and projected distances from the Galactic Center detailed at the end of Sec. II. Readers wishing to rescale these bounds for different dark matter background density models should note that the bound on $\kappa^{2} \alpha_{D} \alpha_{\mathrm{EM}}$ scales as $\frac{1}{\rho_{x}}$. Bounds using gas cloud G1.4 - 1.8 + 87, using the temperature reported in Ref. [5], have been indicated as preliminary; see Sec. VII. A few benchmark masses with $m_{A^{\prime}} \ll \alpha m_{e}$ are shown. The constraints from the Galactic Center gas cloud apply up to coupling values given in Eqs. (14) and (15). The mass range $m_{A^{\prime}} \ll \alpha m_{e}$ is chosen in this figure for ease of comparison with terrestrial experiments (SENSEI [68] and XENON [69]). Other constraints come from the SLAC millicharge search [70] shown in gray, supernova 1987A cooling [71] presented in green, and the stellar cooling [72] shown in red. The constraints on effective relativistic degrees of freedom $N_{\text {eff }}$ from BBN and CMB observations [72] are shown in cyan and purple, respectively. 

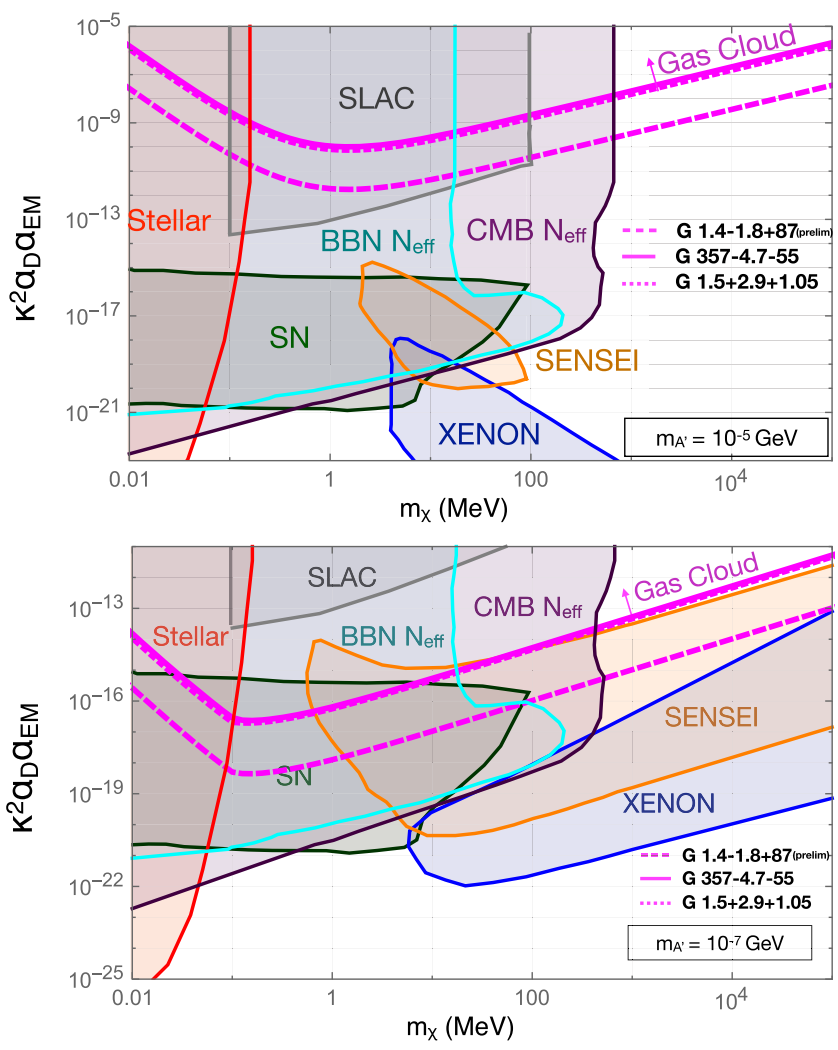

FIG. 4. Constraints on the product of the couplings $\kappa^{2} \alpha_{D} \alpha_{\mathrm{EM}}$ are shown in magenta (regions above each line are excluded), for vector portal dark matter heating of gas clouds with cloud parameters given in Table 1 and local dark matter densities according to a generalized NFW profile and projected distances from the Galactic Center detailed at the end of Sec. II. Readers wishing to rescale these bounds for different dark matter background density models should note that the bound on $\kappa^{2} \alpha_{D} \alpha_{\mathrm{EM}}$ scales as $\frac{1}{\rho_{x}}$. Bounds using gas cloud G1.4-1.8+87, using the temperature reported in Ref. [5], have been indicated as preliminary; see Sec. VII. A few benchmark masses with $m_{A^{\prime}}>\alpha m_{e}$ are shown. We note that constraints from the Galactic Center gas cloud are effective up to coupling values given in Eq. (15). Terrestrial direct detection bounds [68,69] are comparatively weak in this regime. We show the bounds for the two cases of $m_{A^{\prime}}=10^{-7} \mathrm{GeV}$ and $m_{A^{\prime}}=10^{-5} \mathrm{GeV}$. For an explanation of the feature apparent at $m_{\chi}=10^{-4} \mathrm{GeV}$ for $m_{A^{\prime}}=10^{-7} \mathrm{GeV}$, see the text. Other bounds (SLAC [70], Supernova [71], $N_{\text {eff }}$ from $\mathrm{BBN}$ and CMB [72], and stellar constraints [72]) are indicated.

Terrestrial direct detection experiments are also only sensitive to dark photon mediated dark matter with sufficiently weak interactions, so that dark matter passes through the Earth's atmosphere and crust without losing too much of its kinetic energy. As a consequence, such terrestrial experiments are sensitive to small values of the dark-visible photon mixing parameter and dark sector gauge coupling constant. Figure 3 shows the comparison of gas cloud bounds with other astrophysical and terrestrial experiments. One significant astrophysical bound is obtained from Supernova1987A [71] (green region in
Fig. 3), since dark photon mediated dark matter can be produced during the implosion of the nascent neutron star. If the dark matter had left the supernova in appreciable quantities, the supernova would have cooled faster than was observed. For Supernova 1987A, any extra sources of energy loss per unit mass have to be smaller than $10^{19} \mathrm{erg} / \mathrm{g} / \mathrm{s}$ at plasma temperatures of approximately $10 \mathrm{MeV}$ [73]. If the dark matter is too strongly interacting, it will be trapped inside the supernova and cannot contribute to its cooling. Therefore, SN1987A also has an upper limit for the constraint it provides, evident in Figs. 3 and 4 . This bound can be sensitive to the effective mass of dark photon. However, for dark photon masses $m_{A^{\prime}}<T_{\mathrm{SN}} \sim 20 \mathrm{MeV}$, the thermal mass of the dark photon in the hot supernova plasma dominates, and the bounds do not change by varying bare $A^{\prime}$ mass.

Another astrophysical observation that constrains the parameter space comes from red giant helium burning and white dwarf stars based on stellar energy loss $[51,72,74,75]$. Dark matter particles can be produced though an exchange of a dark photon in the stellar interior and leave the star, resulting in a faster cooling rate for these stars. The bounds coming from stellar observations are shown in red in Fig. 3. The effective number of relativistic degrees of freedom during big bang nucleosynthesis (BBN) and cosmic microwave background also provide substantial bounds on the parameter space [72].

Another important bound comes from the electron beam dump experiment at SLAC [70]. This experiment consisted of a $20 \mathrm{GeV}$ electron beam impinging upon a set of fixed aluminum plates. A pair of dark matter particles can be produced via the exchange of an off-shell $A^{\prime}$ : $e^{-} N \rightarrow e^{-} N A^{\prime *} \rightarrow e^{-} N \bar{\chi} \chi$. The dark matter would then traverse through a $179 \mathrm{~m}$ wide hill, followed by $204 \mathrm{~m}$ of air, before scattering off of electrons detected in an electromagnetic calorimeter. We might also consider bounds from production of the dark photon itself. However, if the dark photon is produced on shell and remains stable until reaching the detector, it will leave no detectable signature in this experiment. So long as $m_{A^{\prime}}<$ $2 m_{e}$ and $m_{A^{\prime}}<2 m_{\chi}$, the bounds from the SLAC experiment on our vector portal dark matter model will only arise from the production of dark matter.

Terrestrial direct detection experiments traditionally provide strong bounds on dark matter heavier than a $\mathrm{GeV}$. However, while XENON10 was intended to constrain dark matter-nucleon scattering, it showed sensitivity to single ionized electrons [69] and could thereby bound dark matter-electron interactions for dark matter masses as small as a few mega-electron-volts. New proposals, using XENON10 as a proof of principle, have suggested the usage of semiconductors due to their lower band gap. These will probe even lighter dark matter and also have an enhancement in the event rate for heavier dark matter masses. One such experiment is SENSEI [68], the results of which are shown in Fig. 4. 
At electron ionization experiments like SENSEI and XENON10, the relevant electrons are often bound to atoms. The typical velocity of a bound electron is $v_{e} \sim \alpha_{\mathrm{EM}}$. Thus, there is often a minimum threshold momentum exchange to dislodge the electron and detect dark matter-electron scattering: $q \sim \alpha_{\mathrm{EM}} m_{e}$. As a result, these electron ionization experiments have taken to quoting electron scattering cross sections with the form

$$
\bar{\sigma}_{e}=\frac{8 \pi \kappa^{2} \alpha_{D} \alpha_{\mathrm{EM}} \mu_{\chi e}^{2}}{\left(m_{A^{\prime}}^{2}+q^{2}\right)^{2}}=\frac{8 \pi \kappa^{2} \alpha_{D} \alpha_{\mathrm{EM}} \mu_{\chi e}^{2}}{\left(m_{A^{\prime}}^{2}+\left(\alpha_{\mathrm{EM}} m_{e}\right)^{2}\right)^{2}},
$$

since smaller momentum exchanges than $q=m_{e} \alpha_{\mathrm{EM}}$ would not dislodge an electron. To translate the bounds provided by these experiments, which are given in terms of the above cross section, we must also multiply $\bar{\sigma}_{e}$ by a form factor $|F(q)|^{2}$, which contains the momentum transfer dependence of the interaction. For example, for a pointlike interaction $m_{A^{\prime}} \gg \alpha m_{e}$, the momentum transfer can be neglected, and $F(q)=1$. For interactions with an ultralight mediator, on the other hand, the form factor is $F(q)=\left(\alpha m_{e} / q\right)^{2}$. In Fig. 3, we have only considered $m_{A^{\prime}} \ll \alpha m_{e} \sim 10^{-6} \mathrm{GeV}$.

The comparison of bounds for heavier dark photons $\left(m_{A^{\prime}} \gtrsim \alpha_{\mathrm{EM}} m_{e}\right)$ is presented in Fig. 4. As is evident, there is some parameter space where cold gas clouds provide the prevailing bound. Gas cloud bounds, which are presented with magenta lines, are particularly important for $m_{x} \gtrsim 1 \mathrm{GeV}$. The astrophysical constraints as well as the bounds from SLAC millicharged experiment and SN1987A remain the same. Finally, we note that there are likely to be bounds (derived at some point in the future) from the nonobservation of spectral distortions of the $\mathrm{CMB}$ power spectrum, which complement cold Galactic Center gas cloud bounds on dark matter-electron scattering via a light vector portal mediator [76-78].

\section{STRONGLY INTERACTING AND COMPOSITE DARK MATTER}

There are a number of models which predict a large cross section for dark matter scattering with nuclei. For example, dark matter charged under the Standard Model SU(3) gauge group, i.e., color-charged dark matter [79-81], near-Planck mass dark matter [82], monopole dark matter (e.g., Ref. [83]), and dark matter composed of many constituent states, also known as composite dark matter [84-90].

One substantial advantage cold gas clouds have in searching for heavy dark matter is a large admitted flux of dark matter. The gas clouds observed at the Milky Way Galactic Center reached thermal equilibrium over the course of millions of years and have radii $r_{g} \sim 10 \mathrm{pc}$. This means that requiring a flux $\left(N_{f}\right)$ of at least ten dark matter objects of mass $m_{x}$ pass through the cloud over a million years $\left(t_{g}\right)$, Galactic Center gas clouds are sensitive to dark matter masses up to $m_{x} \sim \pi r_{g}^{2} \rho_{x} v_{x} t_{g} / N_{f}$,

$$
\begin{aligned}
m_{x} \simeq 3 & \times 10^{60} \mathrm{GeV}\left(\frac{r_{g}}{10 \mathrm{pc}}\right)^{2}\left(\frac{\rho_{x}}{10 \mathrm{GeV} / \mathrm{cm}^{3}}\right) \\
& \times\left(\frac{v}{0.001 c}\right)\left(\frac{t_{g}}{10^{6} \mathrm{yrs}}\right)\left(\frac{10}{N_{f}}\right) .
\end{aligned}
$$

For objects so massive-one clear candidate would be primordial black holes-heating by gravitational processes like dynamical friction should be considered. In this study, we restrict our attention to dark matter masses up to approximately $10^{32} \mathrm{GeV}$ and assume that the dark matter primarily couples to baryons via a nongravitational interaction.

The sensitivity of underground experiments [91-99], above-ground searches [100-114], and cosmological surveys $[50,72,76-78,115,116]$ to dark matter's interactions with baryons is summarized in Fig. 5. Bounds are expressed in terms of the per-nucleon scattering cross section, for ease of comparison between experiments, since target nuclei at these experiments vary. For example, argon, fluorine, and xenon are typical nuclear targets at underground direct detection experiments, while oxygen is a predominant nuclear target in ancient mica and Skylab's plastic etch detectors. Our treatment here assumes that dark matter couples equally to protons and neutrons in nuclei. Then, in the case in which dark matter couples directly to nucleons through a "contact" interaction, e.g., the mass of the boson mediating the interaction is much greater than the momentum exchange, the spin-independent dark matter-nucleon interaction is given in terms of the nuclear interaction as [117]

$$
\sigma_{n x}=\left(\frac{\mu_{n x}}{\mu_{N x} A}\right)^{2} \sigma_{N x} \frac{1}{F_{A}^{2}\left(E_{n r}\right)},
$$

where $\mu_{n x} \equiv \frac{m_{x} m_{n}}{m_{x}+m_{n}}, \mu_{N x} \equiv \frac{m_{x} m_{N}}{m_{x}+m_{N}}$ are reduced masses, $m_{n}$ is the nucleon mass, $m_{N}$ is the nuclear mass, $m_{x}$ is the dark matter mass, $A$ is the number of nucleons in the nucleus, and $\sigma_{N x}$ is the dark matter-nuclear scattering cross section. The dark matter-nuclear scattering form factor for a laboratory frame nuclear recoil energy of $E_{n r}$ is given by

$$
F_{A}^{2}\left(E_{n r}\right)=\left(\frac{3 J_{1}(q r)}{q r}\right)^{2} e^{-s^{2} q^{2}}
$$

where the size of the nucleus is approximately $r=$ $\sqrt{r_{n}^{2}-5 s^{2}}$ for nuclear skin depth $s=1 \mathrm{fm}$ and nuclear radius $r_{n}=1.2 A^{1 / 3} \mathrm{fm}, J_{1}$ is the first Bessel function, and the momentum transfer in the scattering interaction is $q=\sqrt{2 m_{N} E_{n r}}$.

Bounds on dark matter interactions with baryons from gas clouds are obtained by requiring that the volumetric rate of dark matter heating via scattering with baryons is less than the volumetric cooling rate of the coldest gas cloud observed in the Galactic Center, VDHR $<$ VCR for gas cloud model $\mathrm{Cl}_{2} 2$ in Table 1 . Specifically, the volumetric heating rate is given by $\mathrm{VDHR}=n_{x} n_{A} \sigma_{N x} v_{x} E_{n r}$, where 
$n_{x}=\rho_{x} / m_{x}$ is the number density of dark matter and $n_{A}$ is the number density of an atomic element in the gas cloud. Summing over contributions from scattering with hydrogen, helium, oxygen, and iron in the gas cloud, with relative mass fractions given assumed to be the solar mass abundances of $\left\{f_{H}, f_{H e}, f_{O}, f_{C}, f_{F e}\right\}=$ $\{0.71,0.27,0.01,0.004,0.0014\}$, this implies the following bound on the per-nucleon scattering cross section,

$$
\sigma_{n x}<\frac{\mathrm{VCR}}{n_{n}}\left(\sum_{A} \frac{f_{A} \mu_{N x} A^{2} n_{x} m_{n}}{\mu_{n x} m_{N}^{2}} \int d^{3} v_{x} v_{x}^{3} F_{A}^{2}\left(E_{n r}\right) B\left(v_{x}, v_{\mathrm{esc}}, y\right)\right)^{-1},
$$

where $B\left(v_{x}, v_{\mathrm{esc}}, y\right)$ is the Maxwell-Boltzmann distribution given in Ref. [3]. In Fig. 5, we find that cold Galactic Center gas clouds provide new bounds on baryonic dark matter interactions, particularly for dark matter masses in excess of $10^{9} \mathrm{GeV}$.

In order to effectively heat the entire gas cloud, dark matter must be able to move with a roughly constant velocity through the gas cloud. For strong enough dark matter interactions, the dark matter will have depleted its kinetic energy so much before reaching the gas cloud interior that it will be incapable of appreciably heating the majority of the gas cloud. Indeed, as is evident in Sec. II and Fig. 1, galactic gas clouds will have relatively hot exteriors. Therefore, if dark matter is substantially slowed in the outer layers of the gas cloud, it would not heat the central region of the gas cloud effectively. Future work may wish to model this explicitly and find how the average and radially distributed gas cloud temperature is affected by dark matter which is substantially slowed as it travels through a gas cloud. In our treatment here, to account for this overburden effect in our Galactic Center gas cloud treatment, we will require that the dark matter retains at least half its kinetic energy after it has traveled to the center of the gas cloud. The formula describing the average depletion of dark matter kinetic energy as it travels through the gas cloud is given by (e.g., Ref. [118])

$$
\frac{E_{f}}{E_{i}}=\prod_{A}\left(1-\frac{4 z m_{N} m_{x}}{\left(m_{N}+m_{x}\right)^{2}}\right)^{n_{A} \sigma_{N x} r}
$$

where $\frac{E_{f}}{E_{i}}$ is the ratio of the final to initial kinetic energy after scattering with the gas cloud, $n_{A} \equiv f_{A} n_{n} \frac{m_{n}}{m_{A}}$ is the number density of nucleus $A, r$ is the distance the dark matter travels in the gas cloud, and $z \in(0,1)$ is a kinematic factor encapsulating the scattering angle; it ranges from zero to 1 for glancing to head-on collisions. The term $\frac{4 z m_{N} m_{x}}{\left(m_{N}+m_{x}\right)^{2}}$ is the fraction of the dark matter's kinetic energy depleted by each scatter. In computing the critical cross section at which dark matter loses half its kinetic energy before reaching the center of the cloud, we take $z=0.5$. The solar metallicity mass fractions given above $\left(f_{A}\right)$, along with the radial depth $r=8 \mathrm{pc}$ and hydrogen density $n_{n} \approx 0.3 \mathrm{~cm}^{-3}$ of gas cloud G1.4 $-1.8+87$, were used to obtain an upper bound on

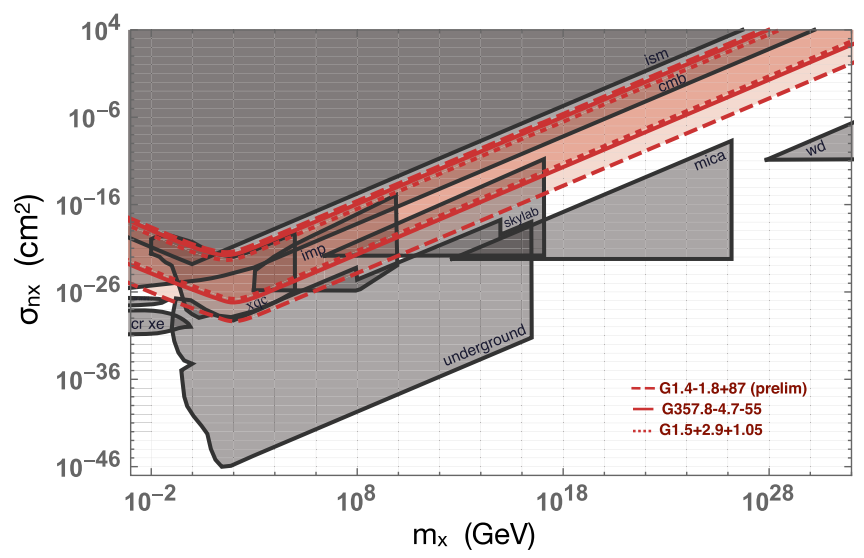

FIG. 5. Existing bounds on the spin-independent dark matternucleon scattering cross section are shown, along with bounds derived from cold gas clouds at the Galactic Center, for dark matter masses ranging from mega-electron-volts to $10^{32} \mathrm{GeV}$, and local dark matter densities according to a generalized NFW profile and projected distances from the Galactic Center detailed at the end of Sec. II. Readers wishing to rescale these bounds for different dark matter background density models should note that the bound on $\sigma_{n x}$ scales as $\frac{1}{\rho_{x}}$. Bounds using gas cloud G1.4 - 1.8 + 87, using the temperature reported in Ref. [5] have been indicated as preliminary; see Sec. VII. Note that cold gas cloud bounds can be accurately extrapolated out to approximately $10^{60} \mathrm{GeV}$; we have truncated the plot for the sake of clarity. From left to right, bottom to top, prior bounds on dark matter-baryon scattering shown in gray are as follows. Cosmic-ray accelerated dark matter is excluded by searches at Xenon1T (cr xe) and MiniBoone (cr mini) [119]. Spectral distortions of the cosmic microwave background exclude stronger dark matter-baryon couplings (cmb) [76,77]. Interstellar gas cooling constraints (ism) were first derived in Ref. [1]. The lower bounds from underground direct detection experiments and particularly XENON1T [91] (underground) are combined with overburden upper bounds which were recently derived and summarized in Ref. [112]. The X-ray Calorimetry Rocket did not observe dark matter events in its calorimeter (xqc) over its 10 min flight [107]. Results from an as-yet-unpublished analysis [103] of Interplanetary Monitoring Platform data [120] are displayed (imp). A charged cosmic-ray search using Skylab's plastic etch detectors was recast for a dark matter bound in Ref. [121]; this analysis (skylab) was later amended in Ref. [101]. A bound from the longevity of white dwarf stars in the Milky Way is also shown (wd) [122]. Nonobservation of tracks in ancient mica excludes dark matter with mass $10^{12}-10^{26} \mathrm{GeV}[99,123,124]$. 
the per-nucleon scattering cross section constrained by Galactic Center gas clouds, shown in Fig. 5.

\section{CONCLUSIONS}

We have used Galactic Center gas clouds to place novel bounds on a number of dark matter scenarios, including ultralight dark photon dark matter, sub-mega electron volts mediated vector portal dark matter, and dark matter that scatters elastically with baryons. After presenting the first detailed study of Galactic Center gas clouds' composition, thermal properties, cooling rates, electron density, metal density, and ionization fractions, we used the derived cooling rates and ionization fraction to set bounds. Remarkably, we have found that Galactic Center gas clouds are excellent detectors for dark matter models spanning the entire range of plausible dark matter masses. Ultralight dark photon dark matter (approximately $10^{-22} \mathrm{eV}$ ), vector portal dark matter (approximately $\mathrm{keV}-\mathrm{TeV}$ mass), and super heavy $\left(\lesssim 10^{60} \mathrm{GeV}\right)$ dark matter that interacts with baryons all lie at the frontier of cold gas cloud detection.

Besides establishing cold gas clouds as excellent dark matter detectors, we have also begun the process of developing accurate models for the cold gas clouds recently discovered at the Galactic Center. The interstellar gas code CLOUDY has been adapted to model cold gas clouds matching the temperature, density, and size of the coldest clouds found near the center of the Milky Way galaxy. Besides validating the estimates of gas cloud cooling used in prior work [3], we explicitly determined the effect of supersolar and subsolar metallicities on their cooling rates, ionization, and the intracloud distribution of each of these quantities. Cold gas cloud modeling will be useful for validating any future cold gas cloud detections of dark matter. In particular, dark matter will alter the thermal properties of gas cloud interiors. In future work, it will be interesting to consider how cold Galactic Center gas clouds' internal thermal structure can be used as a diagnostic of dark matter interactions.

\section{ADDENDUM}

Recently, some work that comments on Galactic Center gas clouds' suitability for setting bounds on dark matter interactions appeared. Regarding gas cloud G1.4 - $1.8+87$, which was reported to have a temperature $T \leq 22 \mathrm{~K}$ in Refs. [5,125], the authors of Ref. [126] have questioned whether G1.4-1.8+87 might not have the cold, $T \leq 22 \mathrm{~K}$, gas core reported in Ref. [5,125]. A number of follow-up observations and studies are planned. While the analysis in Refs. [5,127] indicates G1.4 $-1.8+87$ has a $T \leq 22 \mathrm{~K}$ core with the statistical significance required to satisfy data quality cuts, we have nevertheless indicated all bounds in this document that use
G1.4 $-1.8+87$ as preliminary, pending further investigation as there are concerns that the $22 \mathrm{~K}$ temperature is due to a single-channel fluctuation.

Separately, the authors of Ref. [128] have inquired about the effect of an approximately $200 \mathrm{~km} / \mathrm{s}, T \sim 10^{6} \mathrm{~K}$ Galactic Center wind, hypothesized and modeled in Ref. [5] to explain the distribution of Galactic Center gas clouds near the inner kiloparsec of the Milky Way. Specifically, the authors of Ref. [128] have questioned whether Galactic Center gas clouds are in heating/cooling "equilibrium," given the hypothetical presence of a hot Galactic Center gas wind.

It is important to note that, while a new gas cloud analysis recently presented in Ref. [128] does assume gas cloud heating/cooling equilibrium when setting bounds, the bounds presented in this paper and in our prior work [3] do not assume heating/cooling equilibrium of gas clouds. Rather, as explained in Sec. II and Ref. [3], our bounds rely on gas cloud cooling being a monotonic decreasing function of temperature, for gas clouds with fixed density and temperatures $\lesssim 1000 \mathrm{~K}$. The basic logic is that, observing such a cold gas cloud, regardless of whether it is presently heating or cooling overall, implies a maximum possible historic heating rate for that gas cloud, or else it would not have been capable of cooling to such a low temperature.

This means that, insofar as the density of the gas clouds is approximately constant, one need not assume heating/ cooling equilibrium to trust the bounds presented in this paper. Nevertheless, we find that standard analysis of Galactic Center gas clouds and the hypothetical surrounding wind indicates that the Galactic Center gas clouds used in this study are not disrupted by the wind hypothesized in Ref. [5]. Indeed, this is the explicit conclusion drawn in Ref. [5]. One test for whether gas clouds are disrupted by a surrounding wind is to compare the shock cooling time, which is the time for the cloud to radiate an $\mathcal{O}(1)$ fraction of its kinetic energy in a region shocked by a hot wind, with the cloud crushing time, which is the time for shocks from the surrounding hot wind to propagate through the cold cloud. A number of simulations have determined that the shock cooling time for the clouds in question is approximately 100 years $[5,129]$. On the other hand, the cloud crushing times for the hot wind hypothesized in Ref. [5] are substantially longer, 1-10 Myr, depending on the density of the cloud, indicating minimal internal disruption and allowing for the assumption of stability when modeling the entrained clouds.

Furthermore, the ability to accelerate the entrained clouds to the velocities and galactic radii at which they are observed provides further constraints on cloud disruption processes. In Ref. [130], it is shown that 
simulations which neglect magnetic fields produce cloud shredding timescales over which the clouds cannot be accelerated by galactic winds to their observed velocities. Magnetic fields are therefore likely to be critical in suppressing cloud disrupting processes such as evaporation and turbulent instabilities. The importance of internal magnetic fields in suppressing the disruption of entrained clouds is demonstrated in full magnetohydrodynamic simulations by Ref. [131], while the suppression of hydrodynamic instabilities due to magnetic fields is discussed in Ref. [132]. Therefore, because our bounds do not rely on the assumption of equilibrium, and moreover the literature indicates these clouds are stable even in the presence of the hypothetical hot wind, we think that it is reasonable to derive bounds using cold Galactic Center gas clouds.

\section{ACKNOWLEDGMENTS}

We wish to thank Navid Abbasi, Maxim Pospelov, Nirmal Raj, Farid Taghinavaz, and Aaron Vincent for discussions. J. B. and F. E. thank the CERN theory group for hospitality. J. B. thanks the organizers of the KITP High Energy Physics at the Sensitivity Frontier Workshop; this research was supported in part by the NSF under Grant No. PHY-1748958. Research at Perimeter Institute is supported by the Government of Canada through Industry Canada and by the Province of Ontario through the Ministry of Economic Development \& Innovation. A. B., J. B., and S.S. acknowledge the support of the Natural Sciences and Engineering Research Council of Canada. J. B. thanks the Aspen Center for Physics, which is supported by NSF Grant No. PHY-1066293.
[1] R. Sekhar Chivukula, A. G. Cohen, S. Dimopoulos, and T. P. Walker, Bounds on Halo Particle Interactions From Interstellar Calorimetry, Phys. Rev. Lett. 65, 957 (1990).

[2] B. Qin and X.-P. Wu, Constraints on the Interaction between Dark Matter and Baryons from Cooling Flow Clusters, Phys. Rev. Lett. 87, 061301 (2001).

[3] A. Bhoonah, J. Bramante, F. Elahi, and S. Schon, Calorimetric Dark Matter Detection With Galactic Center Gas Clouds, Phys. Rev. Lett. 121, 131101 (2018).

[4] S. Dubovsky and G. Hernandez-Chifflet, Heating up the galaxy with hidden photons, J. Cosmol. Astropart. Phys. 12 (2015) 054.

[5] N. M. McClure-Griffiths, J. A. Green, A. S. Hill, F. J. Lockman, J. M. Dickey, B. M. Gaensler, and A. J. Green, Atomic hydrogen in a galactic center outflow, Astrophys. J. 770, L4 (2013).

[6] S. De Rijcke, J. Schroyen, B. Vandenbroucke, N. Jachowicz, J. Decroos, A. Cloet-Osselaer, and M. Koleva, New composition dependent cooling and heating curves for galaxy evolution simulations, Mon. Not. R. Astron. Soc. 433, 3005 (2013).

[7] G. J. Ferland, M. Chatzikos, F. Guzmán, M. L. Lykins, P. A. M. van Hoof, R. J. R. Williams, N. P. Abel, N. R. Badnell, F. P. Keenan, R. L. Porter, and P. C. Stancil, The 2017 release cloudy, Rev. Mex. Astron. Astrofis. 53, 385 (2017).

[8] L. Deharveng, M. Peña, J. Caplan, and R. Costero, Oxygen and helium abundances in Galactic Hii regions-II. Abundance gradients, Mon. Not. R. Astron. Soc. 311, 329 (2000).

[9] B. D. Savage and K. R. Sembach, Interstellar abundances from absorption-line observations with the Hubble Space Telescope, Annu. Rev. Astron. Astrophys. 34, 279 (1996).

[10] W. R. J. Rolleston, S. J. Smartt, P. L. Dufton, and R. S. I. Ryans, The Galactic metallicity gradient, Astron. Astrophys. 363, 537 (2000).
[11] W. J. Maciel, and C. Quireza, Abundance gradients in the outer galactic disk from planetary nebulae, Astron. Astrophys. 345, 629 (1999).

[12] J. Dorschner and T. Henning, Dust metamorphosis in the galaxy, Astron. Astrophys. Rev. 6, 271 (1995).

[13] D. C. B. Whittet, Dust in the Galactic Environment (Institute of Physics Publishing, Boca Raton, 1992), p. 306.

[14] P. M. W. Kalberla and J. Kerp, The hi distribution of the milky way, Annu. Rev. Astron. Astrophys. 47, 27 (2009).

[15] A. J. Benson, Galaxy formation theory, Phys. Rep. 495, 33 (2010).

[16] S. De Rijcke, J. Schroyen, B. Vandenbroucke, N. Jachowicz, J. Decroos, A. Cloet-Osselaer, and M. Koleva, New composition-dependent cooling and heating curves for galaxy evolution simulations, Mon. Not. R. Astron. Soc. 433, 3005 (2013).

[17] A. Dalgarno and R. A. McCray, Heating and ionization of hi regions, Annu. Rev. Astron. Astrophys. 10, 375 (1972).

[18] M. G. Wolfire, D. Hollenbach, C. F. McKee, A. G. G. M. Tielens, and E. L. O. Bakes, The neutral atomic phases of the interstellar medium, Astrophys. J. 443, 152 (1995).

[19] S. Bowyer, The cosmic far ultraviolet background, Annu. Rev. Astron. Astrophys. 29, 59 (1991).

[20] C.-A. Faucher-Giguère, A. Lidz, M. Zaldarriaga, and L. Hernquist, A new calculation of the ionizing background spectrum and the effects of He II reionization, Astrophys. J. 703, 1416 (2009).

[21] T. K. Gaisser, The cosmic-ray spectrum: From the knee to the ankle, J. Phys. Conf. Ser. 47, 15 (2006).

[22] M. Amenomori et al., Anisotropy and corotation of galactic cosmic rays, Science 314, 439 (2006).

[23] G. B. Field, D. W. Goldsmith, and H. J. Habing, Cosmicray heating of the interstellar gas, Astrophys. J. 155, L149 (1969). 
[24] C. F. McKee and J. P. Ostriker, A theory of the interstellar medium-Three components regulated by supernova explosions in an inhomogeneous substrate, Astrophys. J. 218, 148 (1977).

[25] L. M. Haffner, R. J. Dettmar, J. E. Beckman, K. Wood, J. D. Slavin, C. Giammanco, G. J. Madsen, A. Zurita, and R. J. Reynolds, The warm ionized medium in spiral galaxies, Rev. Mod. Phys. 81, 969 (2009).

[26] L. Blitz, Y. Fukui, A. Kawamura, A. Leroy, N. Mizuno, and E. Rosolowsky, Giant molecular clouds in local group galaxies, Protostars and Planets V, edited by B. Reipurth, D. Jewitt, and K. Keil (University of Arizona Press, Tucson, 2007), pp. 81-96.

[27] K. M. Ferrière, The interstellar environment of our galaxy, Rev. Mod. Phys. 73, 1031 (2001).

[28] D. Seifried, W. Schmidt, and J. C. Niemeyer, Forced turbulence in thermally bistable gas: A parameter study, Astron. Astrophys. 526, A14 (2011).

[29] G. J. Ferland, R. L. Porter, P. A. M. van Hoof, R. J. R. Williams, N. P. Abel, M. L. Lykins, G. Shaw, W. J. Henney, and P. C. Stancil, The 2013 release of cloudy, Rev. Mex. Astron. Astrofis. 49, 137 (2013).

[30] T. Do, W. Kerzendorf, N. Winsor, M. Støstad, M. R. Morris, J. R. Lu, and A. M. Ghez, Discovery of lowmetallicity stars in the central parsec of the milky way, Astrophys. J. 809, 143 (2015).

[31] M. Ness and K. Freeman, The metallicity distribution of the Milky Way bulge, Pub. Astron. Soc. Aust. 33, e022 (2016).

[32] P. A. M. Van Hoof, J. C. Weingartner, P. G. Martin, K. Volk, and G. J. Ferland, Grain size distributions and photoelectric heating in ionized media, Mon. Not. R. Astron. Soc. 350, 1330 (2004).

[33] J. C. Weingartner, B. T. Draine, and D. K. Barr, Photoelectric emission from dust grains exposed to extreme ultraviolet and X-ray radiation, Astrophys. J. 645, 1188 (2006).

[34] F. Haardt and P. Madau, Radiative transfer in a clumpy universe. IV. New synthesis models of the cosmic UV/X-ray background, Astrophys. J. 746, 125 (2012).

[35] N. Indriolo, T. R. Geballe, T. Oka, and B. J. McCall, $\mathrm{H}_{3}^{+}$ in diffuse interstellar clouds: A tracer for the cosmic-ray ionization rate, Astrophys. J. 671, 1736 (2007).

[36] N. Indriolo and B. J. McCall, Investigating the cosmic-ray ionization rate in the galactic diffuse interstellar medium through observations of $\mathrm{H}^{+3}$, Astrophys. J. 745, 91 (2012).

[37] B. J. McCall, A. J. Huneycutt, R. J. Saykall, T. R. Geballe, N. Djuric, G. H. Dunn, J. Semaniak, O. Novotny, A. AlKhalili, and A. Ehlerding, An enhanced cosmic-ray flux towards $\zeta$ Persei inferred from a laboratory study of the $\mathrm{H}_{3}^{+}-e^{-}$recombination rate, Nature (London) 422, 500 (2003).

[38] U. Maio, K. Dolag, B. Ciardi, and L. Tornatore, Metal and molecule cooling in simulations of structure formation, Mon. Not. R. Astron. Soc. 379, 963 (2007).

[39] A. K. Hernandez, B. P. Wakker, R. A. Benjamin, D. French, J. Kerp, F. J. Lockman, S. O'Toole, and B. Winkel, A low-metallicity molecular cloud in the lower galactic Halo, Astrophys. J. 777, 19 (2013).

[40] Y. Sofue, Rotation curve and mass distribution in the galactic center-From black hole to entire galaxy, Publ. Astron. Soc. Jpn. 65, 118 (2013).
[41] M. Benito, N. Bernal, N. Bozorgnia, F. Calore, and F. Iocco, Particle dark matter constraints: the effect of galactic uncertainties, J. Cosmol. Astropart. Phys. 02 (2017) 007; Erratum, J. Cosmol. Astropart. Phys.06 (2018) E01(E).

[42] A. W. Graham, D. Merritt, B. Moore, J. Diemand, and B. Terzic, Empirical models for dark matter halos. I. Nonparametric construction of density profiles and comparison with parametric models, Astron. J. 132, 2685 (2006).

[43] J. F. Navarro, C. S. Frenk, and S. D. M. White, A universal density profile from hierarchical clustering, Astrophys. J. 490, 493 (1997).

[44] E. Vanhollebeke, M. A. T. Groenewegen, and L. Girardi, Stellar populations in the galactic bulge, arXiv:0903.0946.

[45] B. Holdom, Two U(1)'s and epsilon charge shifts, Phys. Lett. 166B, 196 (1986).

[46] M. Pospelov, A. Ritz, and M. B. Voloshin, Secluded WIMP dark matter, Phys. Lett. B 662, 53 (2008).

[47] M. Pospelov, Secluded U(1) below the weak scale, Phys. Rev. D 80, 095002 (2009).

[48] L. Ackerman, M. R. Buckley, S. M. Carroll, and M. Kamionkowski, Dark matter and dark radiation, Phys. Rev. D 79, 023519 (2009).

[49] N. Arkani-Hamed, D. P. Finkbeiner, T. R. Slatyer, and N. Weiner, A theory of dark matter, Phys. Rev. D 79, 015014 (2009).

[50] S. D. McDermott, H.-B. Yu, and K. M. Zurek, Turning off the lights: How dark is dark matter, Phys. Rev. D 83, 063509 (2011).

[51] S. Davidson, S. Hannestad, and G. Raffelt, Updated bounds on millicharged particles, J. High Energy Phys. 05 (2000) 003.

[52] P. Galison and A. Manohar, Two Z's or not two Z's, Phys. Lett. 136B, 279 (1984).

[53] A. E. Nelson and J. Scholtz, Dark light, dark matter and the misalignment mechanism, Phys. Rev. D 84, 103501 (2011).

[54] P. Arias, D. Cadamuro, M. Goodsell, J. Jaeckel, J. Redondo, and A. Ringwald, WISPy cold dark matter, J. Cosmol. Astropart. Phys. 06 (2012) 013.

[55] P. W. Graham, J. Mardon, and S. Rajendran, Vector dark matter from inflationary fluctuations, Phys. Rev. D 93, 103520 (2016).

[56] M. Pospelov, A. Ritz, and M. B. Voloshin, Bosonic superWIMPs as keV-scale dark matter, Phys. Rev. D 78, 115012 (2008).

[57] J.f A. Dror, K. Harigaya, and V. Narayan, Parametric resonance production of ultralight vector dark matter, Phys. Rev. D 99, 035036 (2019).

[58] R. T. Co, A. Pierce, Z. Zhang, and Y. Zhao, Dark photon dark matter produced by axion oscillations, Phys. Rev. D 99, 075002 (2019).

[59] P. Agrawal, N. Kitajima, M. Reece, T. Sekiguchi, and F. Takahashi, Relic abundance of dark photon dark matter, arXiv: 1810.07188 .

[60] J. Jaeckel and A. Ringwald, The low-energy frontier of particle physics, Annu. Rev. Nucl. Part. Sci. 60, 405 (2010).

[61] D. Feldman, B. Kors, and P. Nath, Extra-weakly interacting dark matter, Phys. Rev. D 75, 023503 (2007). 
[62] D. Feldman, Z. Liu, and P. Nath, The Stueckelberg Z-prime extension with kinetic mixing and milli-charged dark matter from the hidden sector, Phys. Rev. D 75, 115001 (2007).

[63] R. Essig et al., Working Group Report: New Light Weakly Coupled Particles, in Proceedings of the 2013 Community Summer Study on the Future of U.S. Particle Physics: Snowmass on the Mississippi (CSS2013): Minneapolis, MN, USA (2013).

[64] E. Izaguirre, G. Krnjaic, P. Schuster, and N. Toro, Analyzing the Discovery Potential for Light Dark Matter, Phys. Rev. Lett. 115, 251301 (2015).

[65] D. Curtin, R. Essig, S. Gori, and J. Shelton, Illuminating dark photons with high-energy colliders, J. High Energy Phys. 02 (2015) 157.

[66] S. Knapen, T. Lin, and K. M. Zurek, Light dark matter: Models and constraints, Phys. Rev. D 96, 115021 (2017).

[67] T. Piffl et al., The RAVE survey: The galactic escape speed and the mass of the Milky Way, Astron. Astrophys. 562, A91 (2014).

[68] M. Crisler, R. Essig, J. Estrada, G. Fernandez, J. Tiffenberg, M. Sofo haro, T. Volansky, and T.-T. Yu (SENSEI Collaboration), SENSEI: First Direct-Detection Constraints on sub-GeV Dark Matter from a Surface Run, Phys. Rev. Lett. 121, 061803 (2018).

[69] R. Essig, T. Volansky, and T.-T. Yu, New constraints and prospects for sub-GeV dark matter scattering off electrons in xenon, Phys. Rev. D 96, 043017 (2017).

[70] A. A. Prinz et al., Search for Millicharged Particles at SLAC, Phys. Rev. Lett. 81, 1175 (1998).

[71] J. H. Chang, R. Essig, and S. D. McDermott, Supernova 1987A constraints on sub-GeV dark sectors, millicharged particles, the QCD Axion, and an Axion-like Particle, J. High Energy Phys. 09 (2018) 051.

[72] H. Vogel and J. Redondo, Dark radiation constraints on minicharged particles in models with a hidden photon, J. Cosmol. Astropart. Phys. 02 (2014) 029.

[73] G. G. Raffelt, Stars as Laboratories for Fundamental Physics (University of Chicago Press, Chicago, 1996).

[74] F. Brummer and J. Jaeckel, Minicharges and magnetic monopoles, Phys. Lett. B 675, 360 (2009).

[75] S. Davidson and M. E. Peskin, Astrophysical bounds on millicharged particles in models with a paraphoton, Phys. Rev. D 49, 2114 (1994).

[76] C. Dvorkin, K. Blum, and M. Kamionkowski, Constraining dark matter-baryon scattering with linear cosmology, Phys. Rev. D 89, 023519 (2014).

[77] V. Gluscevic and K. K. Boddy, Constraints on Scattering of $\mathrm{keV}-\mathrm{TeV}$ Dark Matter with Protons in the Early Universe, Phys. Rev. Lett. 121, 081301 (2018).

[78] W. L. Xu, C. Dvorkin, and A. Chael, Probing sub-GeV dark matter-baryon scattering with cosmological observables, Phys. Rev. D 97, 103530 (2018).

[79] S. Raby, Gauge mediated SUSY breaking at an intermediate scale, Phys. Rev. D 56, 2852 (1997).

[80] J. Kang and M. A. Luty, Macroscopic strings and 'Quirks' at Colliders, J. High Energy Phys. 11 (2009) 065.

[81] V. De Luca, A. Mitridate, M. Redi, J. Smirnov, and A. Strumia, Colored dark matter, Phys. Rev. D 97, 115024 (2018).
[82] H. Davoudiasl and G. Mohlabeng, GeV-Scale messengers of Planck-scale dark matter, Phys. Rev. D 98, 115035 (2018).

[83] V. V. Khoze and G. Ro, Dark matter monopoles, vectors and photons, J. High Energy Phys. 10 (2014) 061.

[84] J. A. Frieman, G. B. Gelmini, M. Gleiser, and E. W. Kolb, Solitogenesis: Primordial Origin of Nontopological Solitons, Phys. Rev. Lett. 60, 2101 (1988).

[85] A. Kusenko and M. E. Shaposhnikov, Supersymmetric Q balls as dark matter, Phys. Lett. B 418, 46 (1998).

[86] G. Krnjaic and K. Sigurdson, Big Bang Darkleosynthesis, Phys. Lett. B 751, 464 (2015).

[87] W. Detmold, M. McCullough, and A. Pochinsky, Dark nuclei I: Cosmology and indirect detection, Phys. Rev. D 90, 115013 (2014).

[88] E. Hardy, R. Lasenby, J. March-Russell, and S. M. West, Signatures of large composite dark matter states, J. High Energy Phys. 07 (2015) 133.

[89] D. M. Grabowska, T. Melia, and S. Rajendran, Detecting dark blobs, Phys. Rev. D 98, 115020 (2018).

[90] A. Coskuner, D. M. Grabowska, S. Knapen, and K. M. Zurek, Direct detection of bound states of asymmetric dark matter, arXiv:1812.07573.

[91] E. Aprile et al. (XENON Collaboration), First Dark Matter Search Results from the XENON1T Experiment, Phys. Rev. Lett. 119, 181301 (2017).

[92] X. Cui et al. (PandaX-II Collaboration), Dark Matter Results From 54-Ton-Day Exposure of PandaX-II Experiment, Phys. Rev. Lett. 119, 181302 (2017).

[93] D. S. Akerib et al. (LUX Collaboration), Results from a Search for Dark Matter in the Complete LUX Exposure, Phys. Rev. Lett. 118, 021303 (2017).

[94] P. A. Amaudruz et al. (DEAP-3600 Collaboration), First Results from the DEAP-3600 Dark Matter Search with Argon at SNOLAB, Phys. Rev. Lett. 121, 071801 (2018).

[95] C. Amole et al. (PICO Collaboration), Dark Matter Search Results from the PICO-60 $\mathrm{C}_{3} \mathrm{~F}_{8}$ Bubble Chamber, Phys. Rev. Lett. 118, 251301 (2017).

[96] Q. Arnaud et al. (NEWS-G Collaboration), First results from the NEWS-G direct dark matter search experiment at the LSM, Astropart. Phys. 97, 54 (2018).

[97] R. Agnese et al. (SuperCDMS Collaboration), Search for Low-Mass Weakly Interacting Massive Particles with SuperCDMS, Phys. Rev. Lett. 112, 241302 (2014).

[98] J. Bramante, B. Broerman, R. F. Lang, and N. Raj, Saturated overburden scattering and the multiscatter frontier: Discovering dark matter at the Planck mass and beyond, Phys. Rev. D 98, 083516 (2018).

[99] J. Bramante, B. Broerman, J. Kumar, R. F. Lang, M. Pospelov, and N. Raj, Foraging for dark matter in large volume liquid scintillator neutrino detectors with multiscatter events, Phys. Rev. D 99, 083010 (2019).

[100] J. Rich, R. Rocchia, and M. Spiro, A search for strongly interacting dark matter, Phys. Lett. B 194, 173 (1987).

[101] P. Charles McGuire, Low background balloon borne direct search for ionizing massive particles as a component of the dark galactic halo matter, Ph.D. thesis, Arizona University, 1994. 
[102] R. Bernabei et al., Extended Limits on Neutral Strongly Interacting Massive Particles and Nuclearites from NaI(Tl) Scintillators, Phys. Rev. Lett. 83, 4918 (1999).

[103] B. D. Wandelt, R. Dave, G. R. Farrar, P. C. McGuire, D. N. Spergel, and P. J. Steinhardt, Selfinteracting dark matter, in Proceedings of the Sources and Detection of Dark Matter and Dark Energy in the Universe (Springer, Dordrecht, 2000), pp. 263-274.

[104] I. F. M. Albuquerque and L. Baudis, Direct Detection Constraints on Superheavy Dark Matter, Phys. Rev. Lett. 90, 221301 (2003); Erratum, Phys. Rev. Lett. 91, 229903(E) (2003).

[105] G. Zaharijas and G. R. Farrar, A Window in the dark matter exclusion limits, Phys. Rev. D 72, 083502 (2005).

[106] G. D. Mack, J. F. Beacom, and G. Bertone, Towards closing the window on strongly interacting dark matter: Far-reaching constraints from Earth's heat flow, Phys. Rev. D 76, 043523 (2007).

[107] A. L. Erickcek, P. J. Steinhardt, D. McCammon, and P. C. McGuire, Constraints on the interactions between dark matter and baryons from the X-ray quantum calorimetry experiment, Phys. Rev. D 76, 042007 (2007).

[108] C. Kouvaris and I. M. Shoemaker, Daily modulation as a smoking gun of dark matter with significant stopping rate, Phys. Rev. D 90, 095011 (2014).

[109] J. H. Davis, Probing Sub-GeV Mass Strongly Interacting Dark Matter with a Low-Threshold Surface Experiment, Phys. Rev. Lett. 119, 211302 (2017).

[110] R. Dick, Very heavy dark Skyrmions, Eur. Phys. J. C 77, 841 (2017).

[111] M. Shafi Mahdawi and G. R. Farrar, Closing the window on $\sim \mathrm{GeV}$ dark matter, J. Cosmol. Astropart. Phys. 12 (2017) 004.

[112] B. J. Kavanagh, Earth scattering of superheavy dark matter: Updated constraints from detectors old and new, Phys. Rev. D 97, 123013 (2018).

[113] D. Hooper and S. D. McDermott, Robust constraints and novel gamma-ray signatures of dark matter that interacts strongly with nucleons, Phys. Rev. D 97, 115006 (2018).

[114] T. Emken and C. Kouvaris, How blind are underground and surface detectors to strongly interacting dark matter, Phys. Rev. D 97, 115047 (2018).

[115] S. L. Dubovsky, D. S. Gorbunov, and G. I. Rubtsov, Narrowing the window for millicharged particles by CMB anisotropy, Pis'ma Zh. Eksp. Teor. Fiz. 79, 3 (2004) [JETP Lett. 79, 1 (2004)].

[116] T. R. Slatyer and C.-L. Wu, Early-universe constraints on dark matter-baryon scattering and their implications for a global $21 \mathrm{~cm}$ signal, Phys. Rev. D 98, 023013 (2018).

[117] J. D. Lewin and P. F. Smith, Review of mathematics, numerical factors, and corrections for dark matter experiments based on elastic nuclear recoil, Astropart. Phys. 6, 87 (1996).
[118] J. Bramante, A. Delgado, and A. Martin, Multiscatter stellar capture of dark matter, Phys. Rev. D 96, 063002 (2017).

[119] T. Bringmann and M. Pospelov, Novel Direct Detection Constraints on Light Dark Matter, Phys. Rev. Lett. 122, 171801 (2019).

[120] IMP-8 Mission, https://spdf.sci.gsfc.nasa.gov/pub/data/ imp/imp8/documents/archived_website/project.html.

[121] Gl. D. Starkman, A. Gould, R. Esmailzadeh, and S. Dimopoulos, Opening the window on strongly interacting dark matter, Phys. Rev. D 41, 3594 (1990).

[122] P. W. Graham, R. Janish, V. Narayan, S. Rajendran, and P. Riggins, White dwarfs as dark matter detectors, Phys. Rev. D 98, 115027 (2018).

[123] P. B. Price and M. H. Salamon, Search for Supermassive Magnetic Monopoles using Mica Crystals, Phys. Rev. Lett. 56, 1226 (1986).

[124] D. M. Jacobs, G. D. Starkman, and B. W. Lynn, Macro dark matter, Mon. Not. R. Astron. Soc. 450, 3418 (2015).

[125] E. M. Di Teodoro, N. M. McClure-Griffiths, F. J. Lockman, S. R. Denbo, R. Endsley, H. A. Ford, and K. Harrington, Blowing in the milky way wind: Neutral hydrogen clouds tracing the galactic nuclear outflow, Astrophys. J. 855, 33 (2018).

[126] G. R. Farrar, F. J. Lockman, N. M. McClure-Griffiths, and D. Wadekar, Comment on the paper "Calorimetric dark matter detection with galactic center gas clouds," arXiv:1903.12191.

[127] N. M. McClure-Griffiths, J. M. Dickey, B. M. Gaensler, A. J. Green, J. A. Green, and M. Haverkorn, The ATCA HI galactic center survey, Astrophys. J. Suppl. Ser. 199, 12 (2012).

[128] D. Wadekar and G. R. Farrar, First direct astrophysical constraints on dark matter interactions with ordinary matter at very low velocities, arXiv:1903.12190.

[129] J. L. Cooper, G. V. Bicknell, R. S. Sutherland, and J. Bland-Hawthorn, Starburst-driven galactic winds: Filament formation and emission processes, Astrophys. J. 703, 330 (2009).

[130] D. Zhang, T. A. Thompson, E. Quataert, and N. Murray, Entrainment in trouble: Cool cloud acceleration and destruction in hot supernova-driven galactic winds, Mon. Not. R. Astron. Soc. 468, 4801 (2017).

[131] M. McCourt, R. M. O'Leary, A.-M. Madigan, and E. Quataert, Magnetized gas clouds can survive acceleration by a hot wind, Mon. Not. R. Astron. Soc. 449, 2 (2015).

[132] S. Orlando, F. Bocchino, F. Reale, G. Peres, and P. Pagano, The importance of magnetic-field-oriented thermal conduction in the interaction of SNR shocks with interstellar clouds, Astrophys. J. 678, 274 (2008). 